\title{
Numerical Simulation of a High-Lift Configuration with Embedded Fluidic Actuators
}

\author{
Veer N. Vatsa* \\ NASA Langley Research Center, Hampton, VA 23681 \\ Damiano Casalino ${ }^{\dagger}$ \\ Exa GmbH, Curiestrasse 4, D-70563, Stuttgart, Germany \\ John C. Lin $\ddagger$ \\ NASA Langley Research Center, Hampton, VA 23681 \\ and \\ Jason Appelbaum ${ }^{\S}$ \\ Exa GmbH, Curiestrasse 4, D-70563, Stuttgart, Germany
}

\begin{abstract}
Numerical simulations have been performed for a vertical tail configuration with deflected rudder. The suction surface of the main element of this configuration is embedded with an array of 32 fluidic actuators that produce oscillating sweeping jets. Such oscillating jets have been found to be very effective for flow control applications in the past. In the current paper, a high-fidelity computational fluid dynamics (CFD) code known as the PowerFLOW ${ }^{\circledR}$ code is used to simulate the entire flow field associated with this configuration, including the flow inside the actuators. The computed results for the surface pressure and integrated forces compare favorably with measured data. In addition, numerical solutions predict the correct trends in forces with active flow control compared to the no control case. Effect of varying yaw and rudder deflection angles are also presented. In addition, computations have been performed at a higher Reynolds number to assess the performance of fluidic actuators at flight conditions.
\end{abstract}

\footnotetext{
${ }^{*}$ Senior Research Scientist, Computational AeroSciences Branch, Research Directorate; Associate Fellow AIAA

${ }^{\dagger}$ Technical Director, Aerospace, Exa GmbH; Member AIAA

${ }_{\ddagger}^{\ddagger}$ Senior Research Engineer, Flow Physics and Control Branch, Research Directorate; Associate Fellow AIAA

$\S$ Aerospace Applications Engineer, Exa GmbH
} 


\section{Nomenclature}

\begin{tabular}{|c|c|}
\hline AFC & active flow control \\
\hline$A_{\text {nozzle }}$ & total exit area of all actuators \\
\hline$A_{\text {ref }}$ & total projected area of entire vertical stabilizer model \\
\hline & semi-span \\
\hline CFD & computational fluid dynamics \\
\hline$C_{D}$ & drag coefficient, $F_{x} /\left(0.5 \rho_{\infty} V_{\infty}^{2} A_{r e f}\right)$ \\
\hline$C_{Y}$ & side-force coefficient, $F_{y} /\left(0.5 \rho_{\infty} V_{\infty}^{2} A_{r e f}\right)$ \\
\hline$C_{Y o}$ & side-force coefficient for non-actuated case \\
\hline$C_{m u}$ & momentum coefficient, $\frac{A_{\text {nozzle }}}{2 * A_{\text {ref }}} *\left(\frac{V_{j}}{V_{\infty}}\right)^{2}$ \\
\hline$C_{p}$ & pressure coefficient, $\left(p-p_{\infty}\right) /\left(0.5 \rho_{\infty} V_{\infty}{ }^{2}\right)$ \\
\hline $\mathrm{c}$ & chord \\
\hline DDES & delayed detached eddy simulation \\
\hline DNS & direct numerical simulation \\
\hline $\operatorname{deg}$ & degrees \\
\hline $\exp$ & experimental \\
\hline ERA & Environmentally Responsible Aviation \\
\hline$F_{x}$ & $\mathrm{x}$-component of integrated force on vertical stabilizer \\
\hline$F_{y}$ & $y$-component of integrated force on vertical stabilizer \\
\hline $\mathrm{k}$ & turbulence kinetic energy \\
\hline $\mathrm{kg}$ & kilogram \\
\hline LBM & lattice Boltzmann model \\
\hline $\mathrm{lbm}$ & pound mass \\
\hline MAC & mean aerodynamic chord \\
\hline $\mathrm{m}$ & meters \\
\hline NASA & National Aeronautics and Space Administration \\
\hline Rey & Reynolds number based on mean aerodynamic chord \\
\hline $\mathrm{t}$ & time \\
\hline $\mathrm{s}, \mathrm{sec}$ & seconds \\
\hline $\mathrm{V}$ & total velocity \\
\hline VR & variable resolution regions \\
\hline $3-\mathrm{D}$ & three-dimensional \\
\hline$u, v, w$ & Cartesian fluid velocity components \\
\hline$x, y, z$ & Cartesian coordinates \\
\hline$\delta_{R}$ & rudder deflection angle \\
\hline$\beta$ & yaw angle \\
\hline$\rho$ & density \\
\hline$\epsilon$ & dissipation length scale for turbulence model \\
\hline
\end{tabular}

\section{Superscript:}

o

degree

Subscript:
$j$
$\infty$

jet exit quantity
free-stream quantity

\section{Introduction}

There has been a growing emphasis on reducing the fuel burn of commercial aircraft to meet the stringent restrictions on emissions and noise imposed by regulatory agencies. Improving the fuel efficiency of aircraft will help in reducing the emissions, as well as the direct operating costs of airplanes, a long term goal for commercial companies and airlines. A significant effort is being devoted to reduce the noise and fuel burn through research activities at NASA in the Environmentally Responsible Aviation (ERA) program. One area of research under this program is to increase the lift/drag ratio 
and/or to reduce the size of control surfaces on aircraft by making use of active flow control (AFC) devices, which will decrease the overall weight and fuel burn of the aircraft, and hence reduce the emissions for a given payload. The AFC devices have to be designed to maximize performance with the least amount of weight penalty and power requirements. In addition, these devices must have acceptable fault tolerance.

Recent advances in computational fluid dynamics (CFD) methods have made it possible to simulate the effect of AFC devices on major aircraft components, as evidenced by a series of publications in the literature. ${ }^{1-5}$ The paper of Collis et al. ${ }^{4}$ summarizes the various issues encountered in active flow control applications from both theoretical and experimental points of view. In most of these studies, flow control was achieved primarily with synthetic or pulsed-jet actuators. These type of devices invariably consist of moving parts and controllers resulting in increased complexity, which in turn can cause operational problems in flight.

Seifert ${ }^{6}$ examined the characteristics and operational mechanism of several different type of actuators, and suggested criteria for comparing their efficiencies. In subsequent papers, Seifert and his co-workers ${ }^{7-9}$ demonstrated the usefulness of AFC devices for reducing separation and drag on different vehicles, and found the suction and oscillatory blowing actuators to be better suited for their applications.

During the last several years, fluidic actuators have been identified as viable flow control devices for improving the aerodynamic performance of high lift configurations by mitigating separated flow regions. Such devices do not contain any moving parts and rely primarily on the feedback mechanism triggered by the unsteady flow inside the actuators. DeSalvo et al., ${ }^{10}$ Woszidlo and Wygnanski, ${ }^{11}$ and Seele et al. ${ }^{12}$ have examined the performance of such actuators for high-lift configurations. Based on the available research, the fluidic actuators that produce spanwise oscillating or sweeping jets without using any moving components, offer a reliable and efficient mechanism for active flow control applications.

In a recent paper, Vatsa et al. ${ }^{13}$ used CFD simulations to examine the internal and external flow structures created by fluidic actuators operating in quiescent air. Numerical studies were conducted to quantify the effect of geometric shape changes and input conditions on the performance characteristics of such actuators. The computed solutions were shown to compare well with available experimental data for several configurations. In the current paper, we examine the effect of an array of such fluidic actuators embedded in a vertical tail configuration that has been tested experimentally by Seele et al. ${ }^{14}$ The primary objective here is to explore and assess the reliability of the PowerFLOW ${ }^{\circledR}$ CFD code for simulating the effect of fluidic actuators on such configurations that produce large side force (lift) when the rudder is deflected.

The outline of the paper is as follows. In section II, the wind-tunnel model and test conditions are described. Section III and IV focus on the numerical simulation method and setup, respectively. The numerical results and the comparisons with the wind-tunnel measurements are presented in Section V, with emphasis given on steady (time-averaged) loads and the effect of flow control. A numerical characterization of the pulsating flow generated by the actuators is also presented in Section V. Finally, the main outcomes of the present effort and directions for further investigations are summarized in the conclusive Section VI.

\section{Configuration and Test Conditions}

The configuration under consideration here corresponds to the vertical tail (stabilizer) model tested by Seele et al. ${ }^{14}$ at the California Institute of Technology's Lucas Wind Tunnel, ${ }^{15}$ shown schematically in Fig. 1 . The basic section of this model has a NACA 0012 profile. The wing is tapered and has a leading edge sweep of 42 degrees and a $35 \%$ chord rudder (flap). It has a span of 3.5 feet $(1.067 \mathrm{~m})$ and a mean aerodynamic chord (MAC) of 1.765 feet $(0.538 \mathrm{~m})$. We will refer to the wing section up to the hing-line as the main-element, and the flap as the rudder for discussion purposes in this paper. This model has 32 fluidic actuators embedded on the suction surface of the main element distributed over the entire span such that the actuator exits are situated in front of the rudder leading edge. The fluidic actuators used on this model have internally curved geometry, and have been studied experimentally by Woszidlo and Wygnanski ${ }^{11}$ and computationally by Vatsa et al. ${ }^{13}$ Such internally curved actuators that produce sweeping jets were found to be more efficient for flow control applications compared to synthetic jets. Additional details of this configuration and test set up are available in Ref. 14.

The fluidic actuators were supplied with compressed air from a feed tube inserted through the root of the wind-tunnel model. The flow rate of air entering the actuators was controlled by an electronic pressure regulator connected to the feed tube. The ejected mass flow was measured by a flow meter. All 32 actuators were located near the trailing edge of the main element. The actuator \# 1 is positioned closest to the root section, whereas actuator \# 32 is placed near the wing tip. The flow exits the actuators at approximately 10 degree angle relative to the surface of the main element of the vertical tail. 
The nominal free-stream velocity in the tunnel is $40 \mathrm{~m} / \mathrm{sec}$. To minimize Reynolds number and transition effects for such low speed flows, tripping dots were applied at $x / c=5 \%$ on the suction side and $x / c=10 \%$ on the pressure side of the model. Pressure measurements along the chordwise direction are available for this configuration at three different locations corresponding to 40,70 , and $89 \%$ span stations of the main element, as shown schematically in Fig. 2 . Note that the pressure measurements for this configuration are available along lines that are normal to the leading edges of the main element and the rudder sections. Lift (side force) and drag force measurements are also available to quantify the effectiveness of the actuators as control devices for improving the performance of the vertical stabilizer.

\section{Simulation Method}

The numerical simulations presented in this paper were performed using the commercial CFD software PowerFLOW ${ }^{\circledR}$ (version 4.4d), which is based on the three dimensional 19 state (D3Q19) lattice Boltzmann model. The lattice Boltzmann model (LBM) can be derived from the velocity space discretization of the general Boltzmann equation, and it recovers the full unsteady Navier-Stokes equations. ${ }^{16-19}$ The PowerFLOW ${ }^{\circledR}$ code can be used to solve the lattice Boltzmann equation in a DNS mode, where all of the turbulent scales are spatially and temporally resolved. However, for most engineering problems at high Reynolds numbers, the simulations are usually conducted in conjunction with a hybrid turbulence modeling approach where the small scales are modeled using a modified two-equation $k-\epsilon$ turbulence model and larger, energy-containing scales are directly resolved. The turbulence model uses a swirl correction to locally reduce the influence of the modeled eddy viscosity to allow vortical structures to develop and persist without added artificial damping. ${ }^{20-22}$ In addition, a wall model relying on algebraic relations such as the logarithmic law of the wall is used to capture the boundary layer behavior and relax the grid spacing requirement near solid walls. ${ }^{23}$ The law of the wall used in this approach has been modified to include pressure gradient effects. The turbulence modeling approach together with the inherently unsteady nature of the LBM ensures accurate and efficient simulation of the large structures, especially in regions of separated flows and shows conceptual similarities with the DDES approach of Spalart et al. ${ }^{24}$

The lattice Boltzmann equation is solved on embedded Cartesian meshes, which are generated automatically within the flow solver for the configuration under consideration, irrespective of the geometric complexity. Variable resolution (VR) regions can be defined to allow for local mesh refinement of the grid by successive factors of two in each direction. ${ }^{25}$ The PowerFLOW ${ }^{\circledR}$ code scales well on modern computer clusters consisting of thousands of processors, making it ideally suitable for large scale applications. The LBM methodology used here has been extensively validated for a wide variety of applications ranging from academic cases by using $\mathrm{DNS}^{26}$ to industrial flow problems in the fields of aerodynamics, thermal management, and aeroacoustics, see e.g., Refs. 5, 22, 27-29.

\section{Computational setup}

The computational setup used in the present study consists of 11 Variable Resolution (VR) levels, numbered from VR0 to VR10. A sketch of the setup is shown in Fig. 3. The simulation domain is enclosed by top, bottom, and side walls where a slip boundary condition is imposed. A viscous, no-slip condition is imposed on the central patch of the bottom wind tunnel wall surrounding the wing root. The upstream size of this viscous wall patch is such that a prescribed value of the boundary layer thickness is achieved at a selected streamwise location. These computational boundaries are used here to approximate the walls of the Caltech Lucas wind-tunnel. ${ }^{15}$ It should be noted that the region below the mounting plate is ignored in current simulations.

A viscous wall condition is imposed on all of the components that form the vertical stabilizer configuration. The presence of the transition tripping dots at $x / c=5 \%$ and $x / c=10 \%$ on the suction and pressure side of the main element is modeled by prescribing laminar patches, as shown in Fig. 4. Velocity is imposed at the inlet boundary, whereas static pressure is imposed at the outlet boundary. The cross-section of the computational domain is nearly identical to the Caltech Lucas wind tunnel. Some of the VR levels are marked on Figs. 3 and 4 for reference. Several of these VR levels have been obtained by offsetting the surface of the model and by setting some regions around specific parts of the model (tip, leading/trailing edges, fixed/movable gap etc.). The highest VR levels, from VR8 to VR10 are used to model the flow inside the actuators, as shown in Fig. 4. In the no-control case, the VR9 and VR10 in the actuators are reverted to VR8 and the overall number of VR levels is reduced to 9 . The minimum voxel (cubic cell) edge length is $3.39 \mathrm{x} 10^{-5} \mathrm{~m}$ and $1.35 \times 10^{-4} \mathrm{~m}$ for the control and no-control cases, respectively. In the actuated (control) case, 30 grid points (cells) are used along the shortest dimension of any rectangular sectional area along the actuator axis.

Fig. 4 shows a global view of the actuation line at the top of the figure, and a detailed view of the VR setup for one of the actuators at the bottom. All the actuators are connected with a single feed pipe of rectangular cross section. Mass flow rate is prescribed at the inlet section of the feed pipe, close to the root of the stabilizer to replicate the experimental 
set up. The VR setup inside the actuators is such that the finest resolution level is set in the throat region, where the highest velocities and shear stresses are expected. The outlet of the actuator is in a VR9 level, corresponding to 15 cells along the height of the actuator. The resolution is progressively coarsened from VR10 to VR6 in the feed pipe. The coarsest voxel size anywhere in the computational domain is set at $0.035 \mathrm{~m}$ for all the cases.

The PowerFLOW ${ }^{\circledR}$ code uses explicit time marching to solve the governing equations in a time consistent manner. The solution on a given VR level is updated twice as often when compared to the number of solution updates on the next coarser VR level. Thus, the solution on the finest grid is updated most frequently, whereas the solution on the coarsest grid is updated least often to advance the solution in the whole domain to the same physical time. Comparable number of voxels (cells) on coarser grids are therefore less expensive from the computational point of view. The computational cost to advance the solution to the same physical time for one voxel on a coarse VR level is $1 / 2$ the cost for a voxel on the next finer VR level and so on. In other words, each successive coarser level voxel is equivalent to $1 / 2$ voxels of the next finer grid in terms of computational cost. For book-keeping purposes and estimating the computational resources, we use the appropriate weighting factors to sum up the contributions of the voxels from different VR levels for obtaining a fine-grid equivalent voxel count, starting with a weight factor of unity for the finest grid. The computational cost per time-step is therefore directly proportional to the fine-grid voxel count obtained in this manner.

\section{Results}

The computations of the three-dimensional (3-D) flow field for the vertical stabilizer configuration with and without flow control (actuation) have been performed using the PowerFLOW ${ }^{\circledR}$ code. The baseline conditions chosen for this work correspond to zero mass flow (no actuation) at a freestream speed of $40 \mathrm{~m} / \mathrm{sec}$ and a rudder deflection of 30 degrees. The Reynolds number based on the mean aerodynamic chord is 1.36 million. In addition to the no-actuation case $\left(C_{m u}=0\right)$, the experimental data for this configuration is also available for several other values of momentum coefficient. Results from the simulations performed at $C_{m u}=0$ and $0.5 \%$, corresponding to the experimental study of Seele et al. ${ }^{14}$ are presented in this paper. Finally, results showing effect of varying the yaw and rudder deflection angles, as well as the Reynolds number are also included here.

The geometric manipulation and grid generation capability available in the PowerFLOW ${ }^{\circledR}$ software makes it very convenient to conduct parametric studies for complex configurations. For the current configuration, the main element and rudder geometries are imported as individual surfaces. With the available information about the center and axis of rotation, we can easily change the rudder deflection and yaw angle to match the experimental test conditions. In addition, the grid resolution is easily changed via input parameters and the grids are generated automatically by the software.

\section{A. Baseline configuration, $\delta_{R}=30^{\circ}, \beta=0^{\circ}$}

The baseline configuration here is the vertical tail configuration of Seele et al. ${ }^{14}$ with a rudder deflection angle $\left(\delta_{R}\right)$ of $30^{\circ}$, and a yaw angle $(\beta)$ of zero degrees. For no actuation case $\left(C_{m u}=0\right)$ at these geometrical settings, a grid consisting of 441 million voxels distributed among 9 VR regions was used. For the actuated case $\left(C_{m u}=0.5 \%\right)$, the computational region employed two additional VR regions for a total of $11 \mathrm{VR}$ regions resulting in 643 million voxels. The fine-grid equivalent voxel count, as defined in the previous section, are comparable for these cases. However, the finest cell size and time-step for the actuated case was $\frac{1}{4} t h$ compared to the non-actuated case. Therefore, greater number of time-steps are required for the flow-field to settle for the actuated case, resulting in higher computational cost for such cases.

For the actuated cases, the governing equations are solved here to simulate the unsteady flow developing inside the fluidic actuators instead of using approximate surface boundary conditions to simulate the effect of actuators. As observed in Ref. 13, the jet exit velocity and frequency of spanwise oscillations of the exit flow depend primarily on the input mass flow (or gauge pressure) and the geometric details of the actuators. The total mass flow going through the actuators is $0.03159 \mathrm{lbm} / \mathrm{sec}(0.01433 \mathrm{~kg} / \mathrm{sec})$ for $C_{m u}=0.5 \%$.

The computations were run long enough for the integrated forces to settle in time before extracting the surface data. The convergence histories for the drag $\left(C_{D}\right)$, and side force $\left(C_{Y}\right)$ coefficients are shown in Fig. 5. Note that due to the inherent unsteady nature of the flow associated with such configurations, the force coefficients display low level of oscillations even for the non-actuated case after the initial transients are settled. Higher level of oscillations in the force coefficients is observed for the corresponding actuated case due to the presence of bi-stable unsteady flow inside the fluidic actuators. Time-averaging was performed starting at $t=0.25$ seconds to obtain the force coefficients. The computed values of side force $\left(C_{Y}\right)$, and the drag force $\left(C_{D}\right)$ coefficients are compared with the experimental data of Ref. 14 in Table 1. The simulations predict the correct qualitative trends with actuation, although the increment in side force due to actuation is under-predicted. 
To ascertain that the integrated forces reported here for the actuated and non-actuated cases were not affected by differences in grid resolution used for these case, the computations for the baseline configuration without the presence of actuation (flow control) were repeated on a grid identical to the actuated case, which consisted of 11 VR regions, instead of the original 9 VR regions employed for this case. The resulting value of side force $\left(C_{Y}\right)$ coefficient based on this simulation differed from the coarser grid results by $0.03 \%$, whereas the drag force coefficient $\left(C_{D}\right)$ differed by $0.05 \%$. Such difference in results due to grid variation are insignificant from a practical point of view, and the rest of the non-actuated cases were performed on the coarser grids consisting of 9 VR regions for efficiency reasons.

Examination of the velocity field revealed the presence of high subsonic (near sonic) velocities inside the actuators for the $C_{m u}=0.5 \%$ case. Accuracy of the current version of PowerFLOW ${ }^{\circledR}$ code (version 4.4d) is known to deteriorate at higher subsonic speeds, and therefore can adversely affect the results of the actuated cases presented here. Effort is currently under way to repeat the actuation cases with a high subsonic (beta) version of the PowerFLOW ${ }^{\circledR}$ code. $^{-}$

Simulated surface streamline patterns super-imposed over pressure coefficient $\left(C_{p}\right)$ contours were created using time-averaged flow field data. The top view of the streamlines on the suction side of this configuration for the actuated and non-actuated cases are compared in Fig. 6. A flow reversal region starting at the hinge-line is observed on the suction surface of the rudder for both of these cases. The biggest difference in the streamline pattern for these cases on the suction side is observed downstream of the hinge line over the rudder. Whereas, a well defined attachment line is visible along major portion of the rudder suction surface for the non-actuated case, no clear attachment line is visible for the actuated case.

Much less effect of actuation is observed on the pressure side of this configuration, as seen in Fig. 7. A perspective view from the suction side is also presented in Fig. 8, displaying the streamline pattern in the vicinity of wing root section. The footprint of a corner vortex can also be seen in this figure, although a better view of the vortical flow is seen in Fig. 9, which shows the unconstrained (3-D) streamline pattern that develops above the suction surface. Identical seeding for streamlines was used to generate these figures for the actuated and non-actuated cases. Corner vortex near the wing root, and tip vortex near the wing tip are clearly visible in this figure. It appears that these vortices are more tightly wound for the non-actuated case (Fig. 9 (a)). The vortices for the actuated case (Fig. 9 (b)) appear more diffused (weaker) and stretched out along the span direction. Unfortunately, equivalent flow visualization images are not available from experimental work at this time.

A more quantitative assessment of the computed results can be made by examining the surface pressure distributions. The computed chordwise pressure distributions at three spanwise stations, namely inboard (40\%), middle (70\%) and outboard (89\%) stations (see Fig. 2 for schematic of span locations), are presented in Fig. 10. The experimental data corresponding to these test conditions is also shown in this figure. The agreement between the computed and measured pressure distributions for the non-actuated case is quite good, except for the suction surface of the rudder on the inboard station. Although, every effort has been made to model the geometric details of the experimental setup based on the available information, it was impractical to replicate every minute detail of the mounting hardware, especially in the wing root region for the current simulations. There was also some uncertainty regarding the precise details of a thin gap on the suction surface along the spanwise direction in the vicinity of the rudder the hinge line. Such geometrical differences are expected to have most influence on the flow field near the inboard section, and in the vicinity of hinge line. These are precisely the regions where the computed results differ the most from the measured data in Fig. 10.

The pressure distributions obtained for the actuated $\left(C_{m u}=0.5 \%\right)$ case indicate higher suction pressure levels similar to the ones observed in the experimental data, and the computational results are found to be in good agreement with the measurements over most of the main wing section. The suction peaks are under-predicted in the vicinity of the hinge line, which could be attributed to either slight differences in geometry or lack of resolution in the suction surface gap region along the hinge line. Despite these differences, the computed results predict the correct overall trends in pressure distribution with active flow control using fluidic actuators.

\section{B. Rudder deflection angle, $\delta_{R}=40^{\circ}, \beta=0^{\circ}$}

The next configuration under consideration had a value of $\delta_{R}=40^{\circ}$ for the rudder deflection angle compared to the baseline case of $\delta_{R}=30^{\circ}$. The process to set up this case of higher rudder deflection angle was very straight-forward and required changing just one input parameter in the PowerFLOW ${ }^{\circledR}$ case setup of the baseline configuration. Simulations have been performed for these rudder settings at $C_{m u}=0$ and $C_{m u}=0.5 \%$, corresponding to the momentum coefficients used for the baseline configuration. To expedite the computations, these cases were initiated using solutions from the baseline configuration of $\delta_{R}=30^{\circ}$. As for the previous case, computations were run long enough in time to purge the initial transients out before starting the averaging process on flow solutions and integrated forces.

The time-averaged pressure distributions for the $40^{\circ}$ rudder deflection angle at middle and outboard span stations are compared with the baseline case in Figs. 11 and 12, respectively. Results for $C_{m u}=0$ and $C_{m u}=0.5 \%$ are 
presented in these figures, along with the results for the baseline configuration of $\delta_{R}=30^{\circ}$ for comparison purposes. The trends observed in the measured data due to increase in rudder deflection angle are replicated by the computational results for both of these cases. For example, the increase in suction peaks in the leading-edge region of the main element are accurately predicted. Another observation worth noting is the relatively flat $C_{p}$ distribution in the aft region of rudder's suction surface, even with actuation for $\delta_{R}=40^{\circ}$. Such pressure plateaus (also seen in the experimental data) are generally observed in the presence of reverse flow regions. Apparently, actuation with a momentum coefficient of $C_{m u}=0.5 \%$ was not adequate to control the flow separation at the higher rudder deflection angle.

The simulated surface streamline pattern for this case are shown in Figs. 13 (a) and (b) for the non-actuated and actuated cases, respectively. From these figures, it is observed that reverse flow is present on a significant part of the rudder for the deflection angle of $\delta_{R}=40^{\circ}$ at $C_{m u}=0$, similar to the baseline case. Comparing the streamline pattern for this case run with $C_{m u}=0.5 \%$ with the baseline case shown in Fig. 6 (b), significant differences are observed on the rudder surface. Although it is difficult to judge the extent of reverse flow region from these surface streamline patterns alone, the presence of an attachment line downstream of hinge line for the higher deflection angle at $C_{m u}=0.5 \%$ is a definite indication of reverse flow in that region.

Table 1. Comparison of force coefficients

\begin{tabular}{|c|c|c|c|c|c|c|c|c|}
\hline$\delta_{R}(\mathrm{deg})$ & $\beta(\mathrm{deg})$ & $C_{m u}(\%)$ & $C_{Y}(\exp )$ & $C_{Y}$ (CFD) & $C_{Y}-C_{Y_{0}}$ (exp) & $C_{Y^{-}} C_{Y_{0}}$ (CFD) & $C_{D}$ (exp) & $C_{D}$ (CFD) \\
\hline 30.0 & 0.0 & 0.0 & 0.780 & 0.8540 & 0.000 & 0.0000 & 0.112 & 0.1019 \\
\hline 30.0 & 0.0 & 0.5 & 0.919 & 0.9273 & 0.139 & 0.0733 & 0.106 & 0.0942 \\
\hline 30.0 & 7.5 & 0.0 & 1.140 & 1.2784 & 0.000 & 0.0000 & 0.163 & 0.1690 \\
\hline 30.0 & 7.5 & 0.5 & 1.227 & 1.3042 & 0.087 & 0.0258 & 0.160 & 0.1543 \\
\hline 40.0 & 0.0 & 0.0 & 0.918 & 1.0134 & 0.000 & 0.0000 & 0.163 & 0.1591 \\
\hline 40.0 & 0.0 & 0.5 & 0.983 & 1.0471 & 0.065 & 0.0337 & 0.159 & 0.1556 \\
\hline
\end{tabular}

\section{Yaw angle variation, $\delta_{R}=30^{\circ}, \beta=7.5^{\circ}$}

The next configuration under consideration was obtained from the baseline configuration by increasing the yaw angle, $\beta$ from $0^{\circ}$ to $7.5^{\circ}$. This yawed case corresponds to one of the configurations tested by Seele et al. ${ }^{14}$ The value of rudder deflection angle, $\delta_{R}$, was kept at $30^{\circ}$ for this series, which corresponds to the rudder deflection of the baseline configuration. Simulations have been performed at these rudder settings with $C_{m u}=0$ and $C_{m u}=0.5 \%$. Once again, the time-averaging process was initiated after the integrated forces started settling in time.

The time-averaged pressure distributions for the $7.5^{\circ}$ yaw angle at middle and outboard span stations are compared with the baseline case in Figs. 14 and 15, respectively. The measured pressure distributions are also presented in these figures, along with the computational results for the baseline configuration $\left(\beta=0^{0}\right)$ for comparison purposes. The trends observed in the measured data due to yaw angle variation are replicated by the computational results for both the non-actuated and actuated cases. For example, the increase in suction peaks in the upstream region of the main element are accurately predicted. A small pressure plateau near $\mathrm{x} / \mathrm{c}=0.2$ is observed in the computed results for the yawed cases, a feature that may also be present in the measured data, but is not obvious due to sparseness of the experimental data. Another observation worth noting is the relatively flat $C_{p}$ distribution in the aft region of rudder's suction surface, even for the case with actuation. Based on the pressure distributions, it is apparent that actuation with a momentum coefficient of $C_{m u}=0.5 \%$ was not adequate to control the flow separation for the yawed configuration.

The simulated surface streamline pattern for the yawed configuration are shown in Figs. 16 for the non-actuated and actuated cases, along with pressure contours. Looking at the pressure levels, it is clear that much higher suction pressure levels are obtained in the leading edge region of the main element over the entire span for both, actuated and nonactuated cases, compared to the baseline configuration (see Fig. 6). For the yawed case with actuation $\left(C_{m u}=0.5 \%\right.$ ), a attachment line is observed in the streamline pattern downstream of hinge line on the rudder. This is indicative of flow reversal in that region and most probably produces smaller suction peaks and pressure plateau on the rudder, as seen in Figs. 14 and 15.

\section{High Reynolds number, Rey $=23 \times 10^{6}$}

The geometric parameters chosen for the next case correspond to the baseline rudder settings, i.e., a rudder deflection angle $\left(\delta_{R}\right)$ of $30^{\circ}$ and a yaw angle $(\beta)$ of $0^{\circ}$. However, the Reynolds number based on the mean aerodynamic chord of 
the main section was set to 23 million, which is typical for a vertical stabilizer in flight. Simulations have been performed for this configuration at $C_{m u}=0$ and $C_{m u}=0.5 \%$, corresponding to the momentum coefficients used for the baseline configuration.

The time-averaged pressure distributions for this case at the middle and outboard span stations are compared with the baseline case in Figs. 17 and 18, respectively. Results for $C_{m u}=0$ and $C_{m u}=0.5 \%$ are presented in these figures, along with the computational results and experimental data for the baseline case for comparison. Experimental data at the higher Reynolds number is currently not available for quantitative assessment at these conditions.

It is instructive to examine the trends in computational results due to increase in Reynolds number. For example, the increase in suction peaks in the leading-edge region of the main element for the non-actuated case due to increase in Reynolds number are comparable to what was observed by increasing the rudder deflection angle from $\delta=30^{\circ}$ to $\delta=40^{\circ}$ (see Figs. 11 and 12). For the actuated case $\left(C_{m u}=0.5 \%\right.$ ), a much larger increase is observed in pressure levels on the suction surface, which in turn produces higher side force (see Table 2) with increase in Reynolds number. A reduction in the drag coefficient is also observed with increase in Reynolds number. Similar trends in forces due to increase in Reynolds number were observed by König et al. ${ }^{30}$ for a high-lift configuration. Based on these results, it appears that fluidic actuators have the potential to significantly enhance the performance of vertical stabilizers under flight conditions.

Table 2. Effect of Reynolds number on force coefficients

\begin{tabular}{|c|c|c|c|c|c|c|c|}
\hline$\delta_{R}(\operatorname{deg})$ & $\beta(\operatorname{deg})$ & $C_{m u}(\%)$ & Rey x $10^{-6}$ & $C_{Y}(\exp )$ & $C_{Y}$ (CFD) & $C_{D}(\exp )$ & $C_{D}(\mathrm{CFD})$ \\
\hline 30.0 & 0.0 & 0.0 & 1.36 & 0.780 & 0.8540 & 0.112 & 0.1019 \\
\hline 30.0 & 0.0 & 0.0 & 23.00 & n.a. & 0.9607 & n.a. & 0.1002 \\
\hline 30.0 & 0.0 & 0.5 & 1.36 & 0.919 & 0.9273 & 0.106 & 0.0942 \\
\hline 30.0 & 0.0 & 0.5 & 23.00 & n.a. & 1.1543 & n.a. & 0.0870 \\
\hline
\end{tabular}

\section{E. Flow structure inside the actuator}

To get a better understanding of the flow structure developing inside the actuator and its impact on the control surface, an instantaneous view of the velocity magnitude field for one of the actuators of the baseline configuration $\left(\delta_{R}=30^{\circ}, \beta=\right.$ $0^{\circ}$ ) run with $C_{m u}=0.5 \%$ is presented in Fig. 19. The micro-jets issued from the actuator nozzle can be seen merging with the flow over the rudder in the vicinity of actuator exits. These frames were generated using the instantaneous velocity field at two different time steps to demonstrate the sweeping (oscillating) nature of the jets. The flow inside the actuator in Fig. 19 (a) appears to be leaning towards the right side of the section, whereas the flow at a later time (Fig. 19 (b)) is leaning the other way. We believe that the ability of fluidic actuators to produce sweeping jets oscillating in spanwise direction that can energize much larger flow regions compared to steady jets issuing from same nozzles fed with identical mass flow rate, contributes to the higher efficiency of these devices for flow control applications, as verified by the experimental work of Seele et al. ${ }^{12,14}$

\section{Concluding Remarks}

Numerical simulations have been performed using the PowerFLOW ${ }^{\circledR}$ CFD code for a vertical tail configuration with deflected rudder. This configuration has an array of fluidic actuators embedded on the suction surface of the main element in proximity of the trailing edge. Such actuators have been found to be very effective for flow control applications in the past. In the current approach, the entire flow field associated with this configuration, including the flow inside the actuators is simulated. The resulting solutions for force coefficients and surface pressure distributions are compared with available experimental data for several rudder settings simulated with and without actuation. Reasonably good agreement is obtained with the measured data for the baseline configuration $\left(\delta_{R}=30^{\circ}, \beta=0^{0}\right)$, and simulated results predict the correct qualitative trends in forces with active flow control at these settings. The simulations under-predicted the increase in side force due to actuation compared to the experimentally measured levels.

Effect of varying yaw and rudder deflection angles are also examined in this paper, and simulations are shown to predict the experimentally observed trends in integrated forces and surface pressure distributions. Even though the experimental data is available only at moderate wind-tunnel Reynolds numbers, results from computations performed at a higher Reynolds number typical of flight conditions are also presented here to assess the performance of fluidic actuators at representative flight conditions. Significant increase in side force coefficient is predicted at higher Reynolds 
number with actuation, thus demonstrating the effectiveness of fluidic actuators at flight conditions.

Future work should focus on re-computing the actuation cases with the newly developed high-subsonic version of the PowerFLOW ${ }^{\circledR}$ code to assess the effect on the aerodynamic forces due to a more accurate simulation of the flow in the vicinity of actuator throat, where flow approaches sonic conditions. Higher deflection and yaw angle cases should also be simulated with higher levels of $C_{m u}$ to determine the suitability of these actuators for controlling flow separation at such conditions. Another area of future research would be to investigate the feasibility of formulating suitable boundary conditions to simulate the effect of such actuator devices on the external flow field in order to improve the computational efficiency.

\section{Acknowledgments}

This work was supported by NASA's Fundamental Aerodynamics and Integrated Systems Research Programs through the Environmentally Responsible Aviation Project. The authors would like to acknowledge the support provided by Roman Seele of California Institute of Technology for providing the experimental data and geometric details of the configuration used in this work. The first author would also like to express his gratitude to Prof. Israel Wygnanski of the University of Arizona for many helpful discussions regarding flow physics associated with fluidic actuators. He would also like to acknowledge the support provided by Mr. Scott Brynildsen of Vigyan Inc. for the geometric modeling of the configurations examined here.

\section{References}

${ }^{1}$ Shmilovich, A. and Yadin, Y., "Flow Control for the Systematic Buildup of High-Lift Systems," Journal of Aircraft, Vol. 45, No. 5, SeptemberOctober 2008, pp. 1680-1688.

${ }^{2}$ Shmilovich, A. and Yadin, Y., "Flow Control Techniques for Transport Aircraft," AIAA Journal, Vol. 49, No. 3, March 2011, pp. 489-502.

${ }^{3}$ Rumsey, C., Gatski, T., Sellers III, W., Vatsa, V., and Viken, S., "Summary of the 2004 Computational Fluid Dynamics Validation Workshop on Synthetic Jets," AIAA Journal, Vol. 44, No. 2, 2006, pp. 194-207.

${ }^{4}$ Collis, S., Joslin, R., Seifert, A., and Theofilis, V., "Issues in active flow control: theory, control, simulation and experiment," Progress in Aerospace Sciences, Vol. 40, No. 4-5, 2004, pp. 237-289.

${ }^{5}$ Brès, G., Fares, E., Williams, D., and Colonius, T., "Numerical Simulations of the Transient Flow Response of a 3D, Low-Aspect-Ratio Wing to Pulsed Actuation," AIAA Paper 2011-3440, June 2011.

${ }^{6}$ Seifert, A., "Closed-loop Active flow control systems: Actuators," Notes on Numerical Fluid Mechanics and Multidisciplinary Design (NNFM), Vol. 95, 2007, pp. 85-102.

${ }^{7}$ Seifert, A., Stalnov, O., Sperber, D., Arwatz, G., Palei, V., David, S., Dayan, I., and Fono, I., "Large Trucks Drag Reduction Using Active Flow Control," AIAA Paper 2008-0743, January 2008.

${ }^{8}$ Arwatz, G., Fono, I., and Seifert, A., "Suction and Oscillatory Blowing Actuator Modeling and Validation," AIAA J., Vol. 40, No. 5, 2008, pp. 1007-1017.

${ }^{9}$ Wilson, J., Schatzman, D., Arad, E., Shtendel, T., and Seifert, A., "Active Separation Control Appled to an Axis-symmetric Body," AIAA Paper 2012-0072, January 2012

${ }^{10}$ DeSalvo, M., Whalen, E., and Glezer, A., "High-Lift Enhancement using Fluidic Actuation,” AIAA Paper 2010-0863, January 2010.

${ }^{11}$ Woszidlo, R. and Wygnanski, I., "Parameters Governing Separation Control with Sweeping Jet Actuators," AIAA Paper 2011-3172, June 2011.

${ }^{12}$ Seele, R., Graff, E., Gharib, M., Taubert, L., Lin, J., and Wygnanski, I., "Improving Rudder Effectiveness with Sweeping Jet Actuators," AIAA Paper 2012-3244, June 2012.

${ }^{13}$ Vatsa, V., Koklu, M., Wygnanski, I., and Fares, E., "Numerical Simulation of Fluidic Actuators for Flow Control Applications," AIAA Paper 2012-3239, June 2011.

${ }^{14}$ Seele, R., Graff, E., Lin, J., and Wygnanski, I., "Performance Enhancement of a Vertical Tail Model with Sweeping Jet Actuators," AIAA Paper 2013-0411, January 2013

${ }^{15} \mathrm{http}: / /$ windtunnel.caltech.edu, last accessed, March 2014.

${ }^{16}$ Chen, H., Chen, S., and Matthaeus, W., "Recovery of the Navier-Stokes Equations Using a Lattice-gas Boltzmann Method," Phys. Rev. A, Vol. 45, 1992, pp. 5339-5342.

${ }^{17}$ Chen, H., Teixeira, C., and Molvig, K., "Digital Physics Approach to Computational Fluid Dynamics, Some Basic Theoretical Features," Intl. J. Mod. Phys. C, Vol. 8, No. 4, 1997, pp. 675-684.

${ }^{18}$ Chen, S. and Doolen, G., "Lattice Boltzmann Method for Fluid Flows," Ann. Rev. Fluid Mech., Vol. 30, January 1998, pp. 329-364.

${ }^{19}$ Shan, X., Yuan, X., and Chen, H., "Kinetic Theory Representation of Hydrodynamics: A Way Beyond the Navier-Stokes Equation," J. Fluid Mech., Vol. 550, March 2006, pp. 413-441.

${ }^{20}$ Chen, H., Kandasamy, S., Orszag, S., Shock, R., Succi, S., and Yakhot, V., "Extended Boltzmann Kinetic Equation for Turbulent Flows," Science, Vol. 301, No. 5633, 2003, pp. 633-636.

${ }^{21}$ Chen, H., Orszag, S., Staroselsky, I., and Succi, S., "Expanded Analogy between Boltzmann Kinetic Theory of Fluid and Turbulence," J. Fluid Mech., Vol. 519, November 2004, pp. 301-314.

${ }^{22}$ Fares, E. and Nölting, S., "Unsteady Flow Simulation of a High-Lift Configuration using a Lattice Boltzmann Approach,” AIAA Paper 2011869 , January 2011.

${ }^{23}$ Teixeira, C., “Incorporating Turbulence Models into the Lattice-Boltzmann Method,” Intl. J. Mod. phys. C, Vol. 9, December 1998, pp. 11591175 . 
${ }^{24}$ Spalart, P., Deck, S., Shur, S., Strelets, M., and Tavin, A., "A New Version of DES Resistant to Ambiguous Grid Densities," Theoretical and Computational Fluid Dynamics, Vol. 20, No. 3, 2006, pp. 181-195.

${ }^{25}$ Chen, H., "Volumetric Formulation of the Lattice Boltzmann Method for Fluid Dynamics: Basic Concept," Phys. Rev. E, Vol. 58, September 1998, pp. 3955-3963.

${ }^{26}$ Li, Y., Shock, R., and Chen, H., "Numerical Study of Flow Past an Impulsively Started Cylinder by Lattice Botzmann Method," J. Fluid Mech., Vol. 519, November 2004, pp. 273-300.

${ }^{27}$ Fares, E., "Unsteady Flow Simulation of the Ahmed Reference Body using a Lattice Boltzmann Approach," Comput. Fluids, Vol. 35, No. 8-9, 2006, pp. 940-950.

${ }^{28}$ Brès, G., Freed, D., Wessels, M., Nöelting, M., and Pérot, F., "Flow and Noise Predictions for Tandem Cylinder Aeroacoustic Benchmark," Physics of Fluids, Vol. 24, No. 3, March 2012, http://dx.doi.org/10.1063/1.3685102.

${ }^{29}$ Casalino, D., Ribeiro, A., and Fares, E., "Facing Rim Cavities Fluctuation Modes," Journal of Sound and Vibration, Vol. 333, No. 13, 2014, pp. 2812-2830.

${ }^{30}$ König, B., Fares, E., and Nöelting, S., "Lattice-Boltzmann Flow Simulations for the HiLiftPW-2,” AIAA Paper 2014-0911, January 2014. 


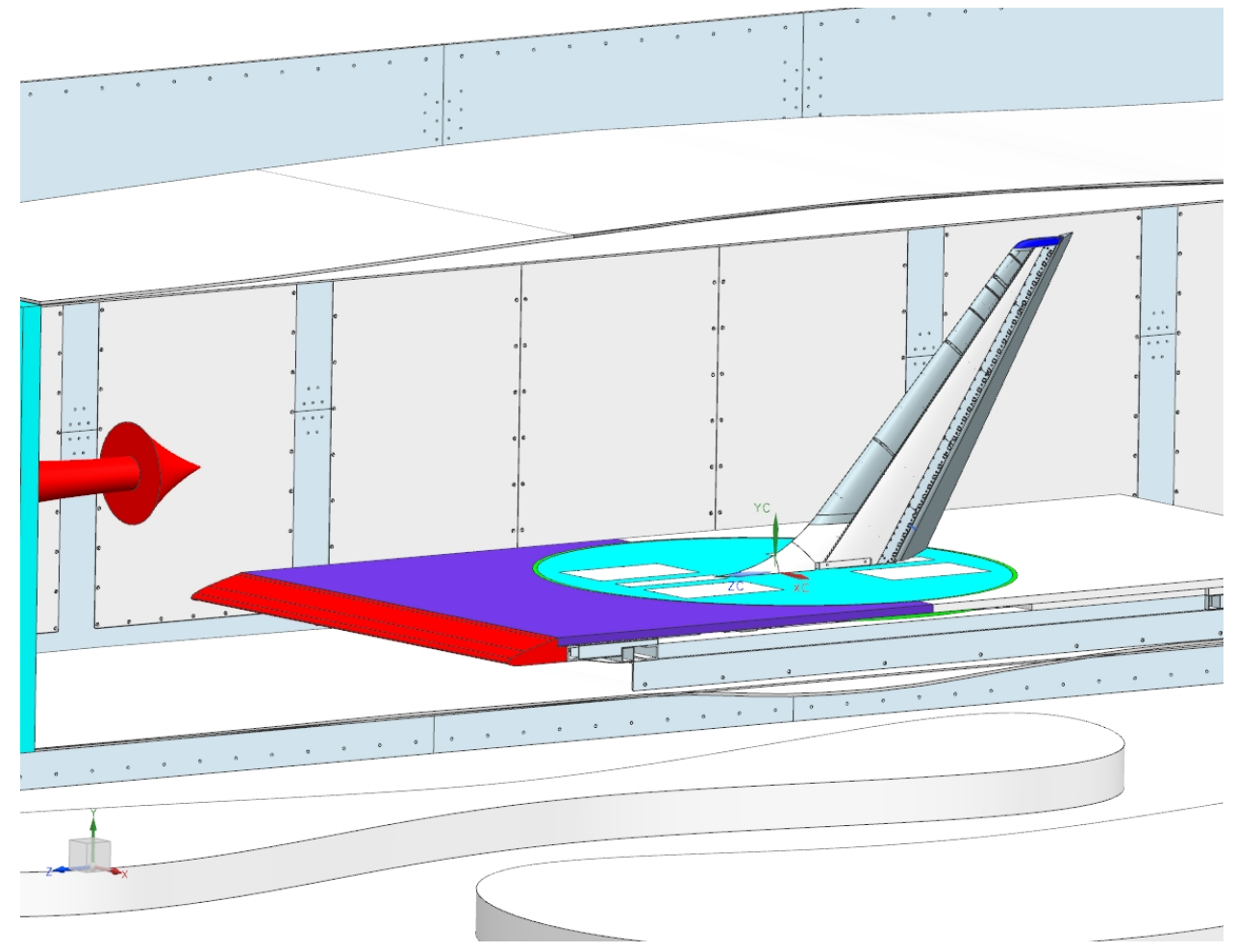

Figure 1. Vertical tail setup in Caltech Lucas wind tunnel.

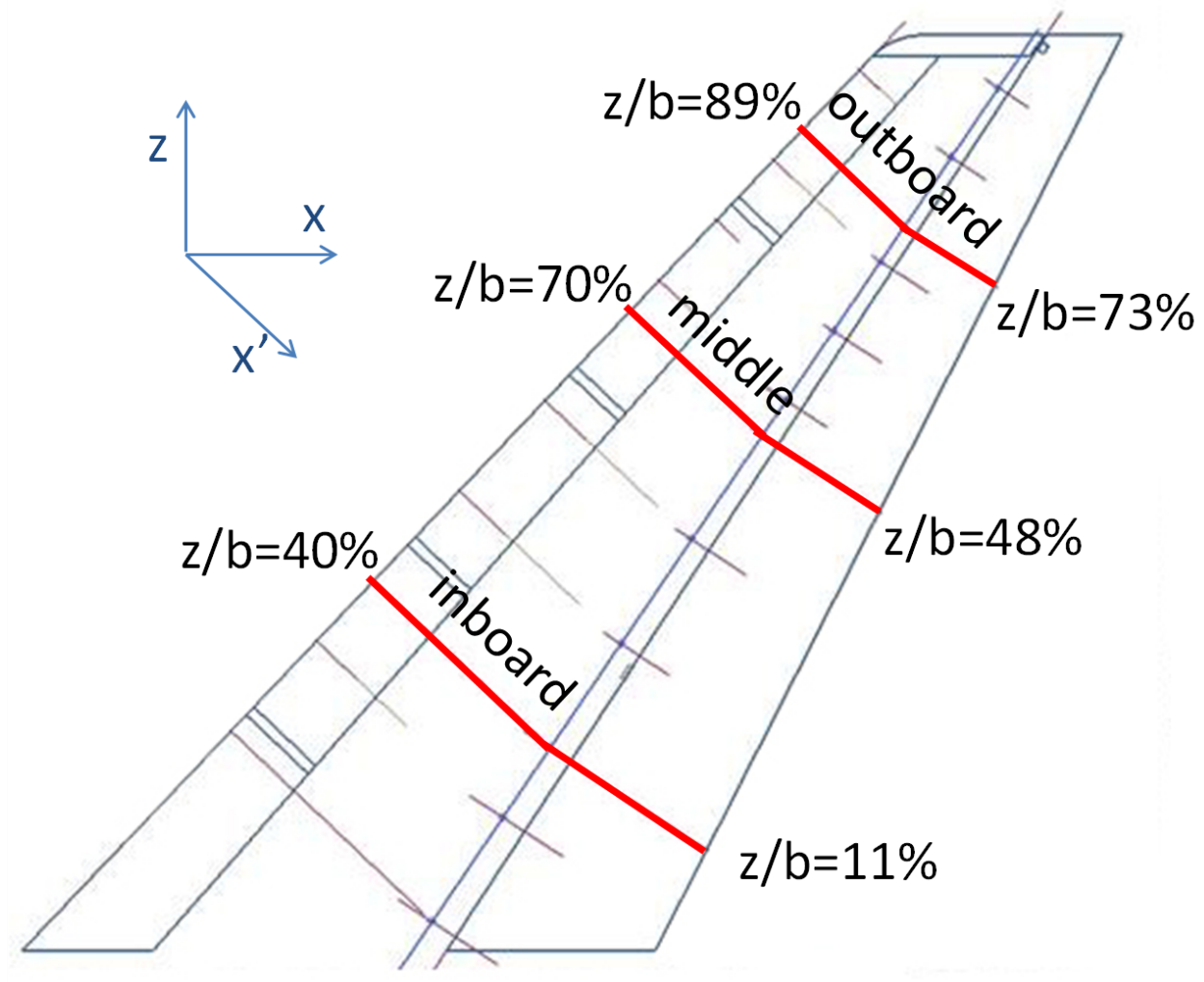

Figure 2. Schematic of chordwise pressure tap rows. 


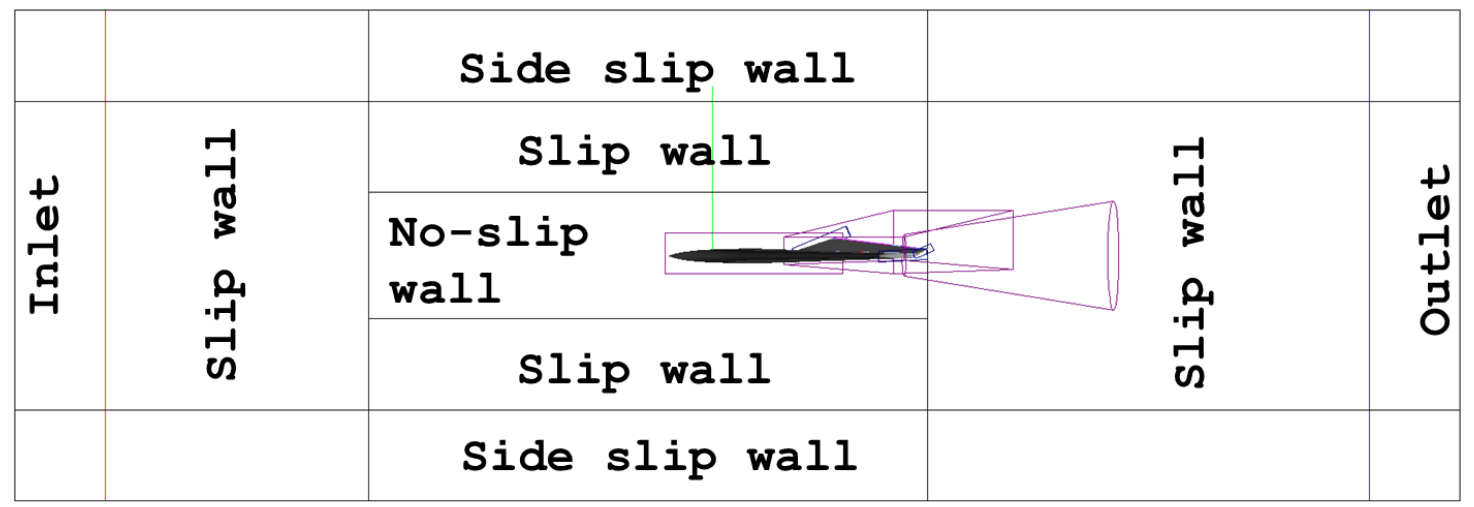

L.

(a) side view

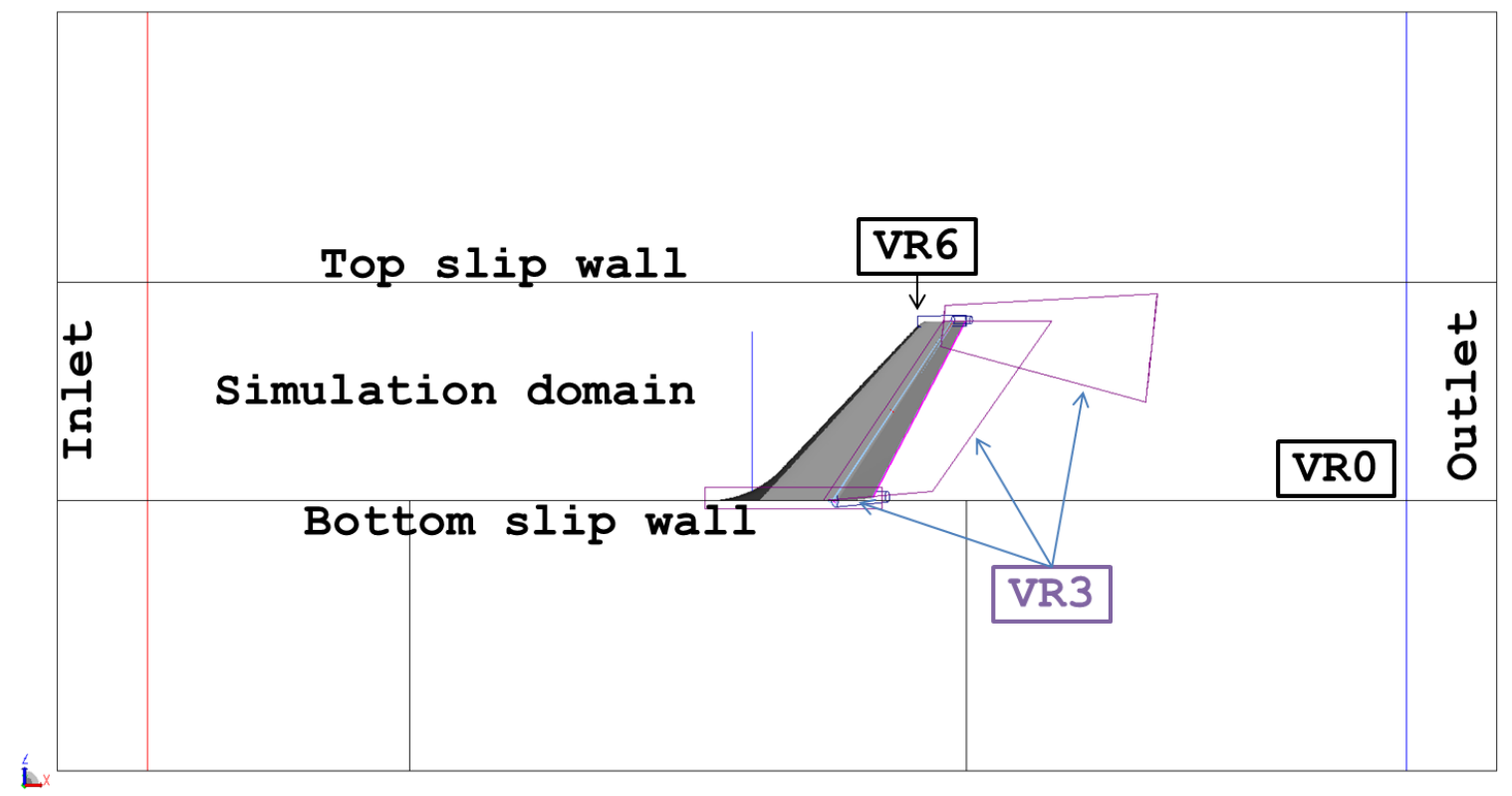

(b) top view

Figure 3. Computational setup. 


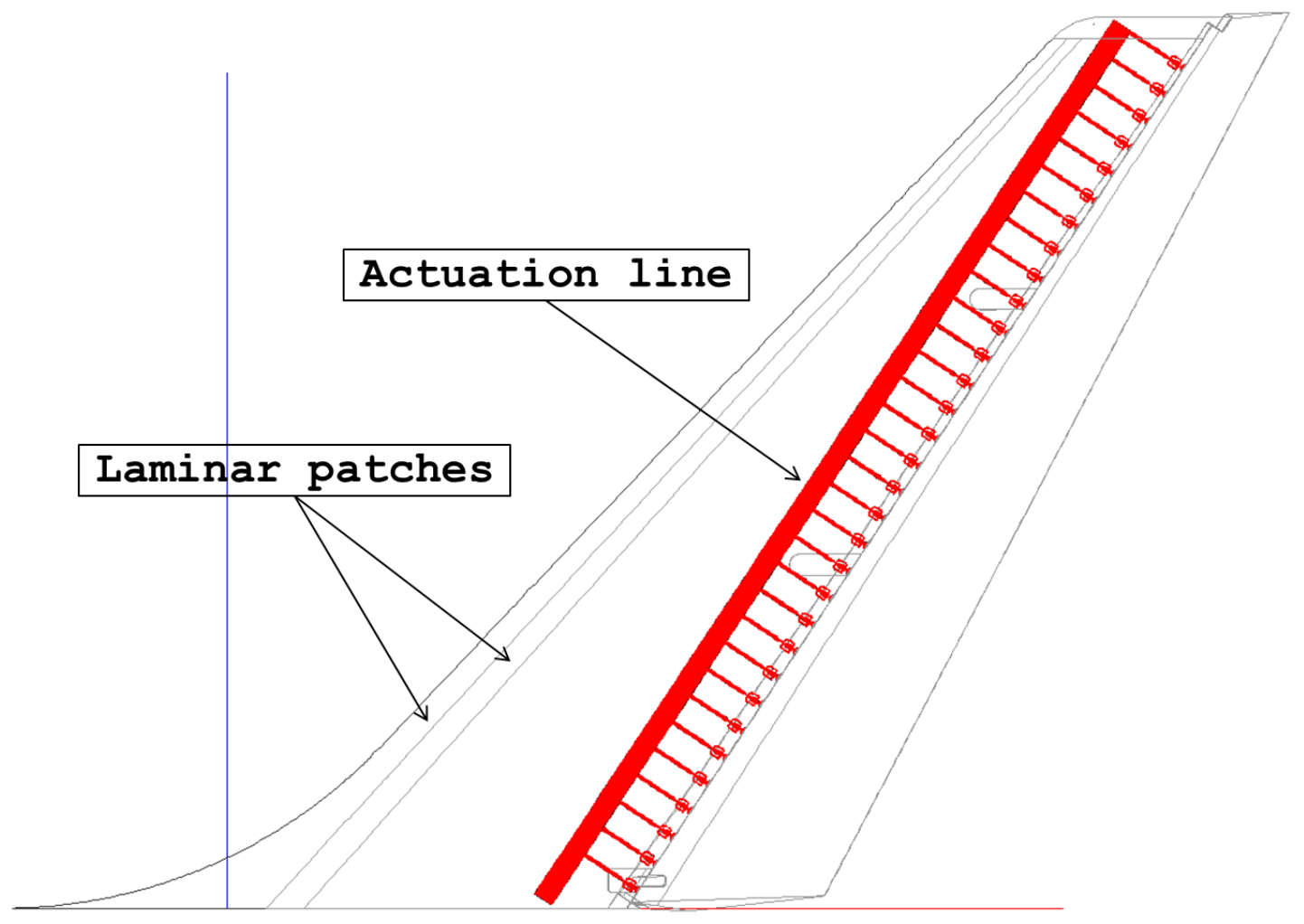

(a) actuation line

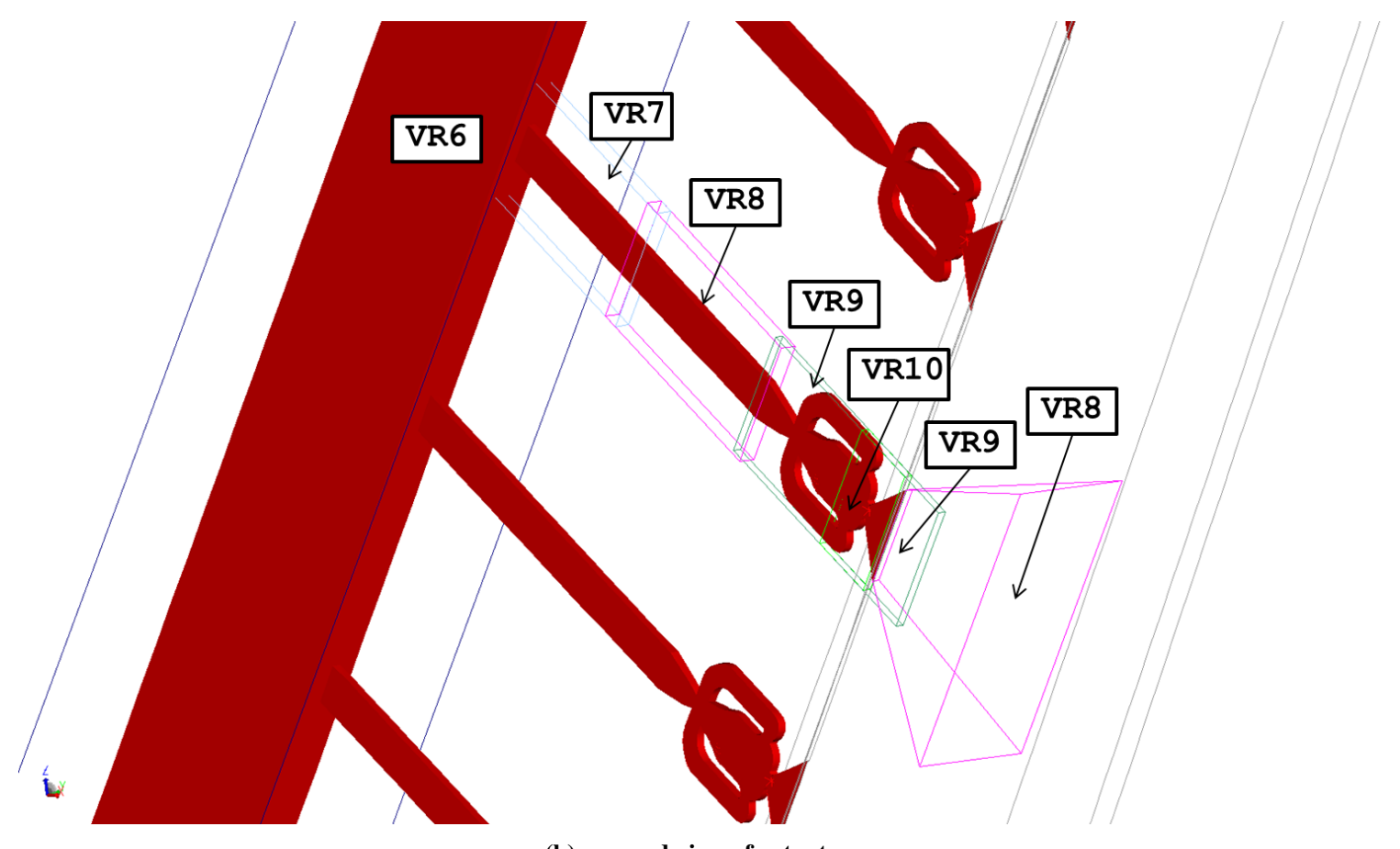

(b) zoomed view of actuators

Figure 4. Actuator setup. 


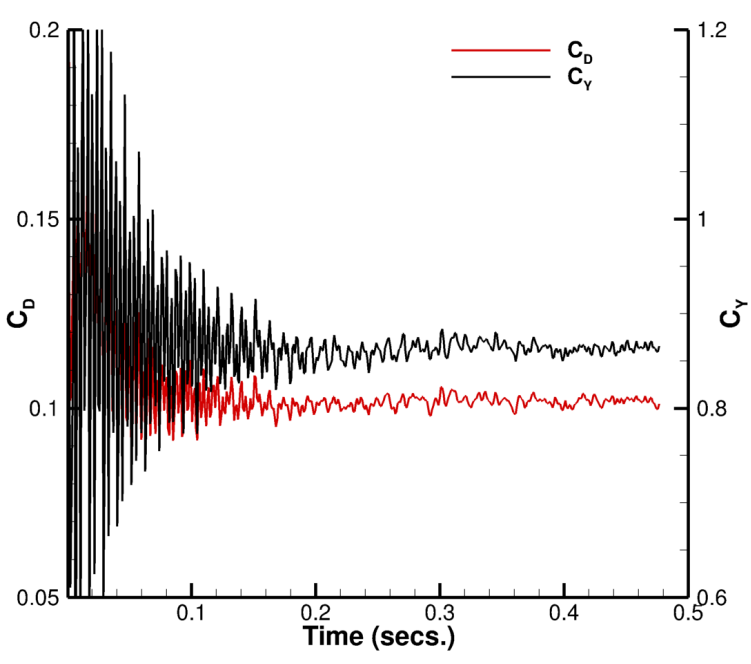

(a) $C_{m u}=0.0 \%$

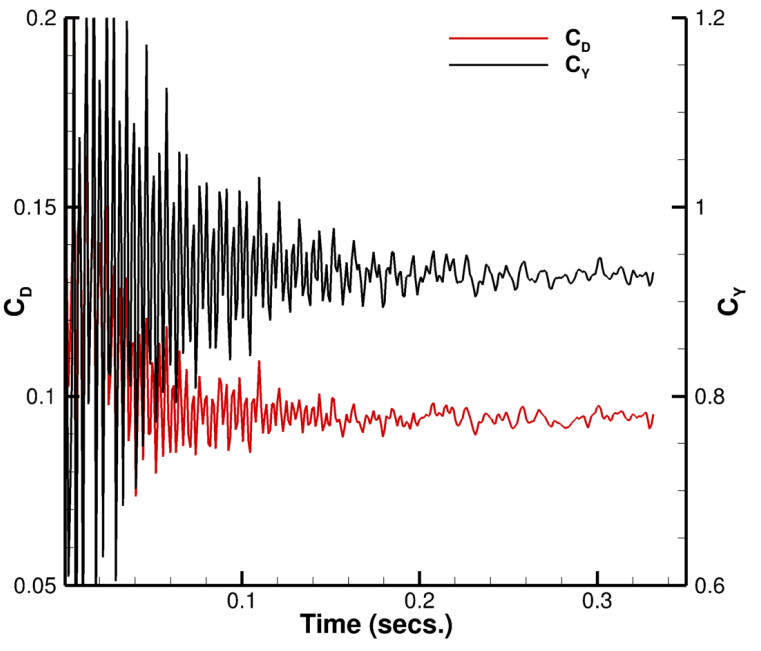

(b) $C_{m u}=0.5 \%$

Figure 5. Convergence history of force coefficients for baseline case. 

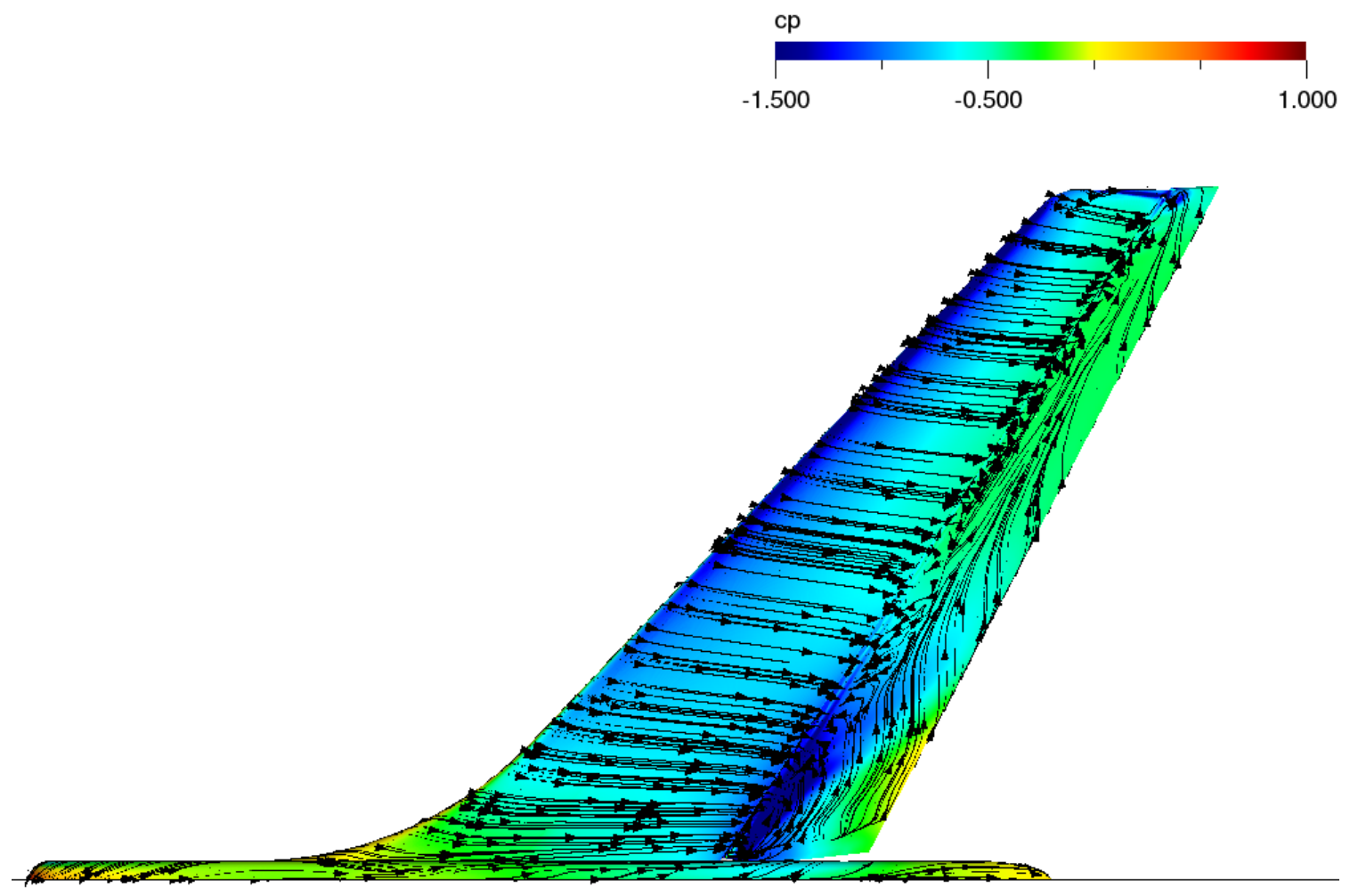

(a) $C_{m u}=0.0 \%$

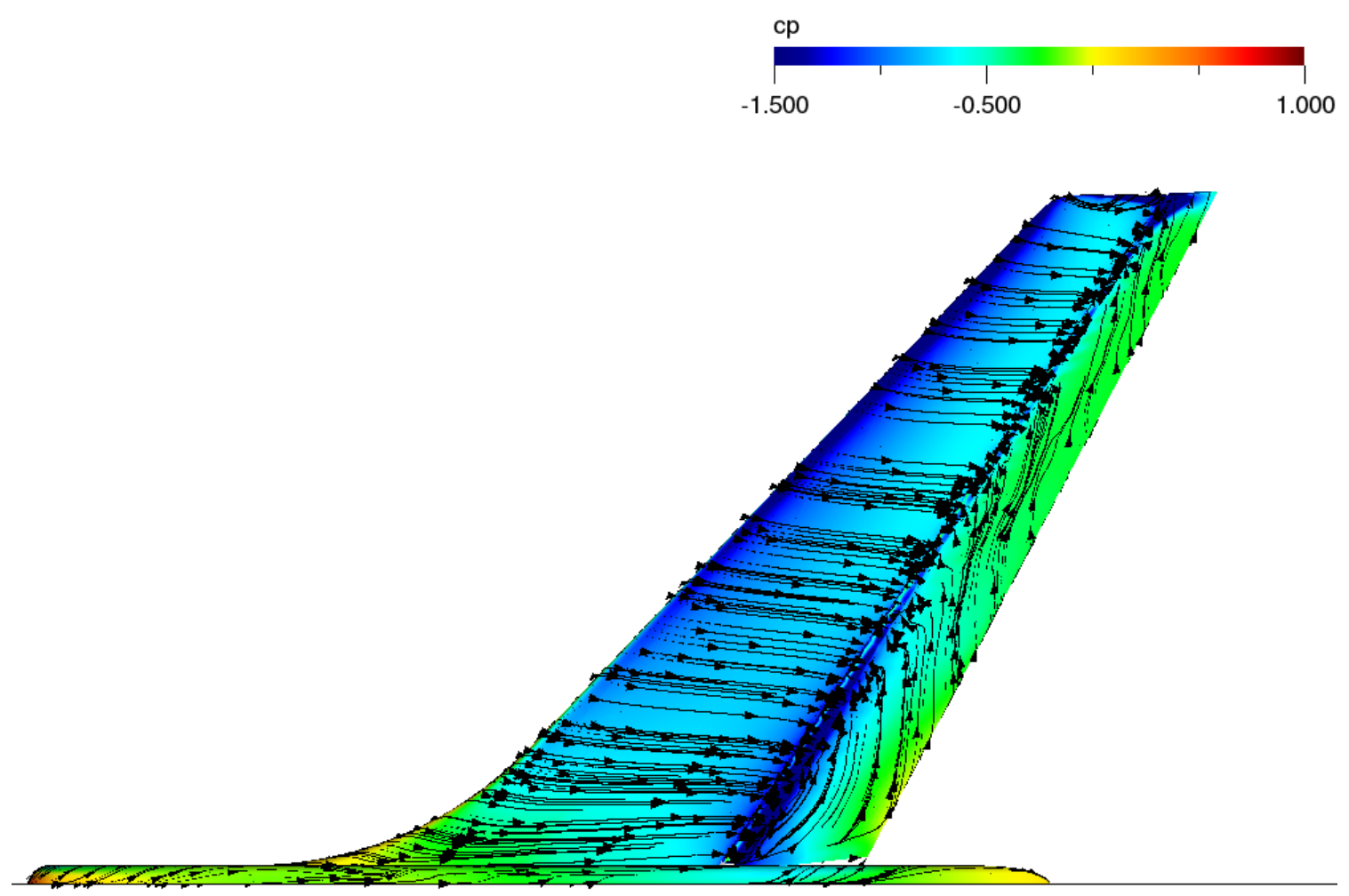

(b) $C_{m u}=0.5 \%$

Figure 6. Top view of simulated suction surface streamlines for baseline configuration. 

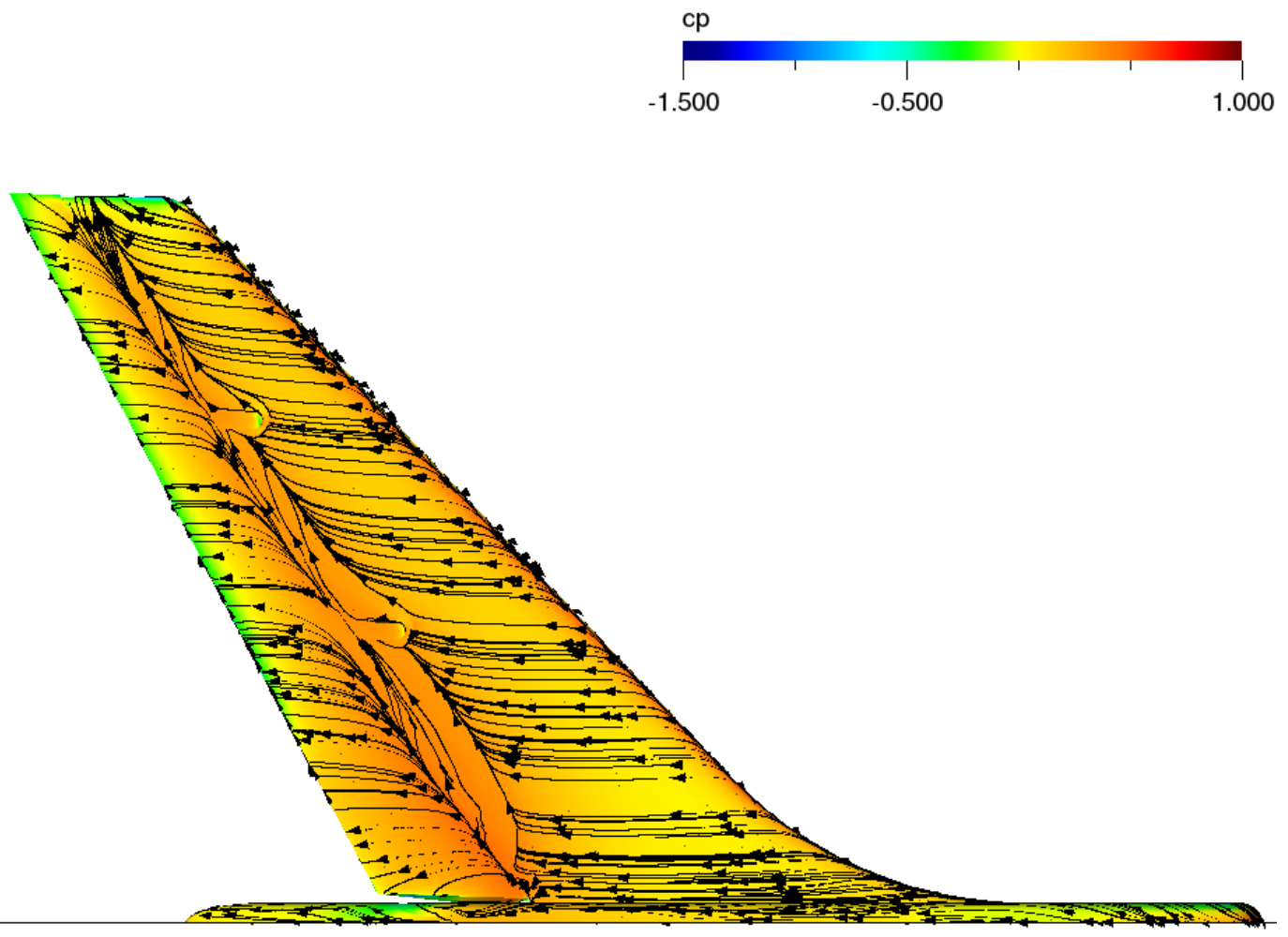

(a) $C_{m u}=0.0 \%$
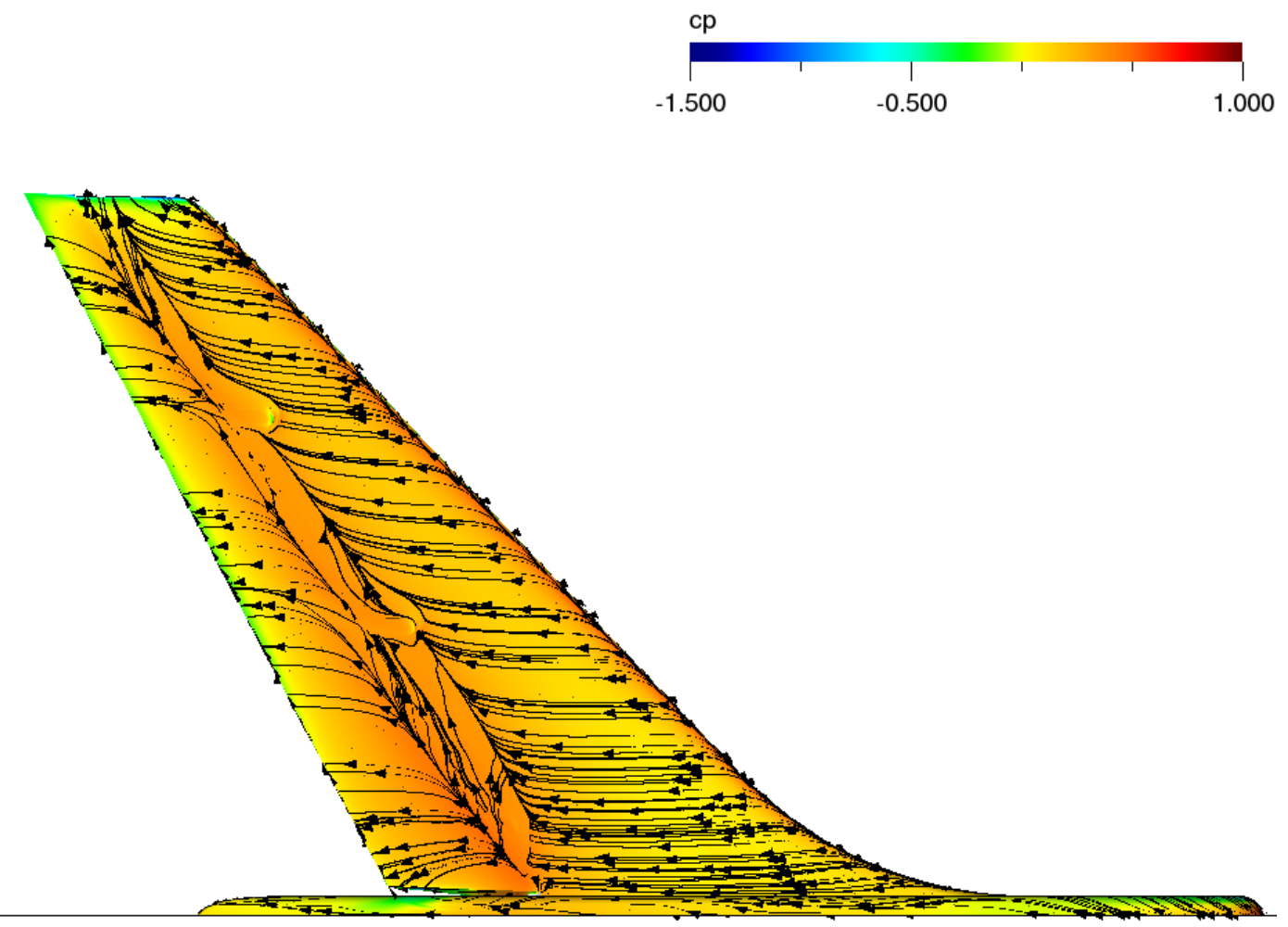

(b) $C_{m u}=0.5 \%$

Figure 7. Simulated pressure surface streamline pattern for baseline configuration. 


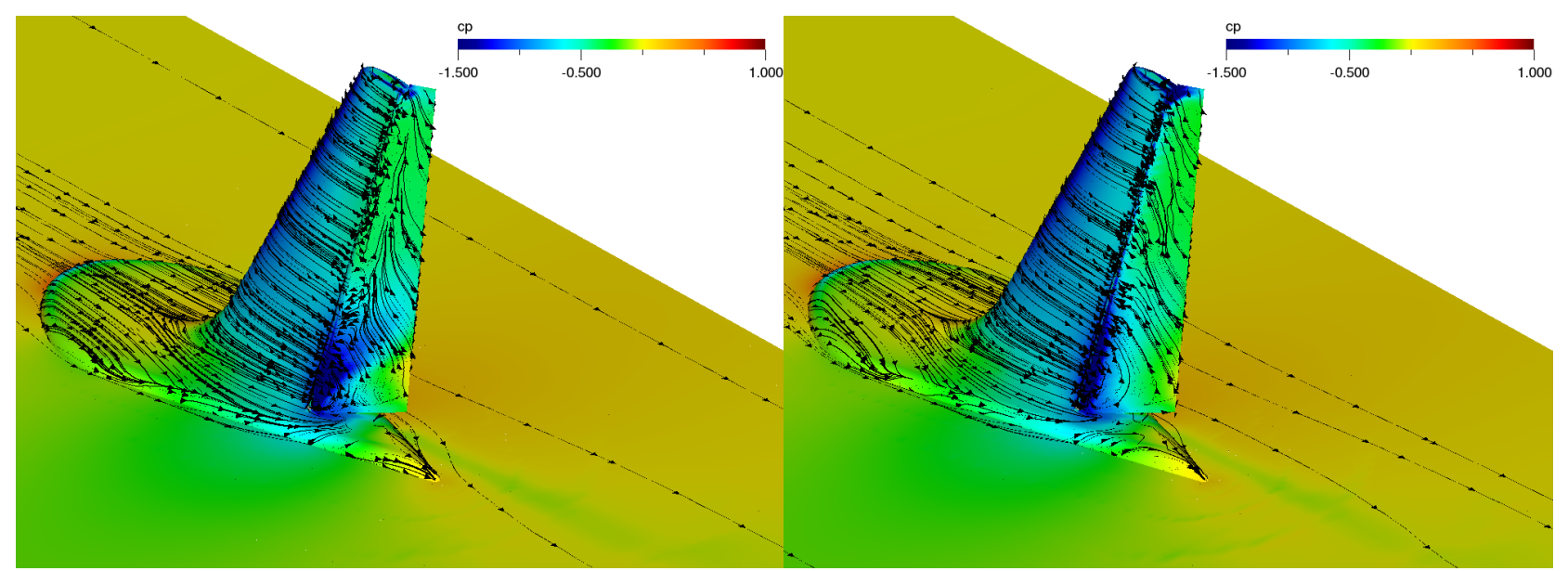

(a) $C_{m u}=0.0 \%$

(b) $C_{m u}=0.5 \%$

Figure 8. Perspective view of simulated suction surface streamlines for baseline configuration.

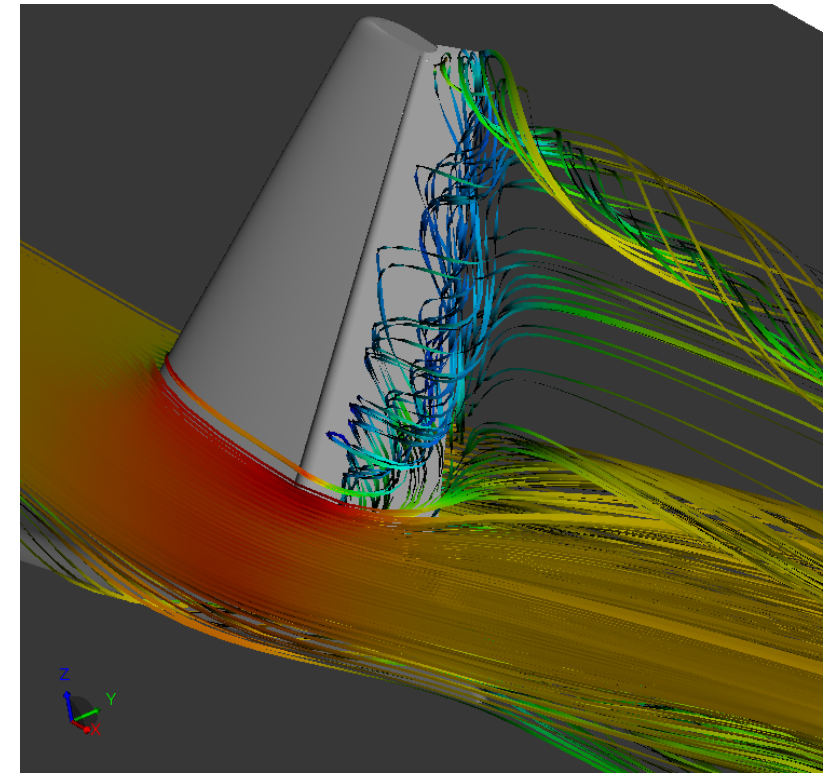

(a) $C_{m u}=0.0 \%$

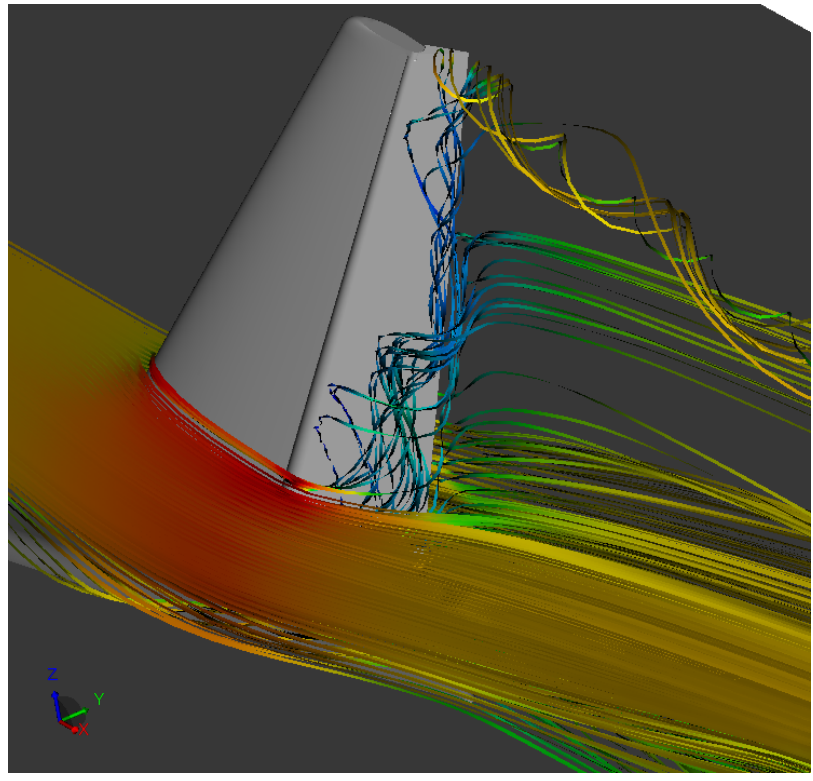

(b) $C_{m u}=0.5 \%$

Figure 9. Simulated 3-D streamlines over suction surface for baseline configuration. 


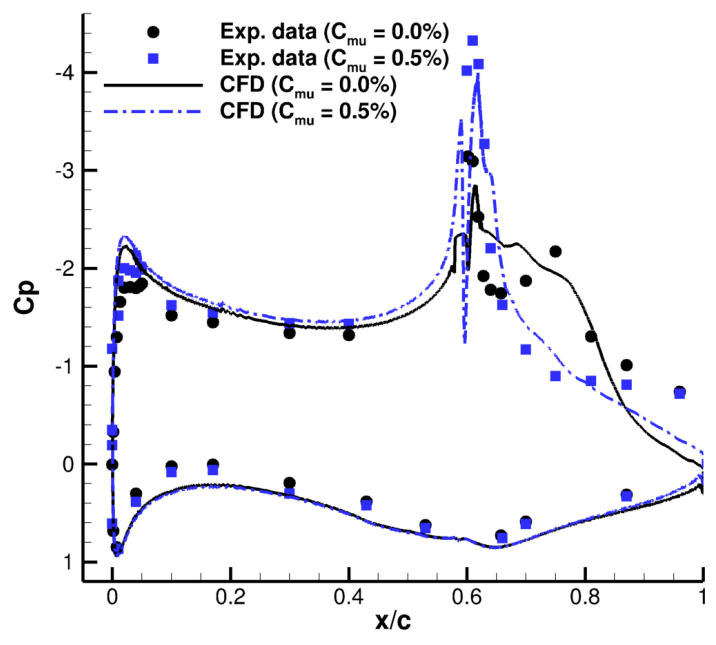

(a) inboard, $z / b=40 \%$

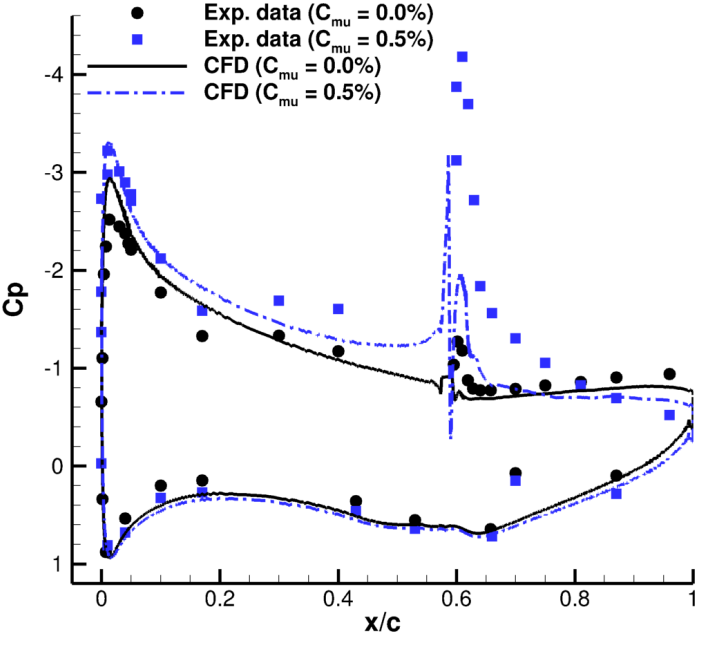

(b) middle, $z / b=70 \%$

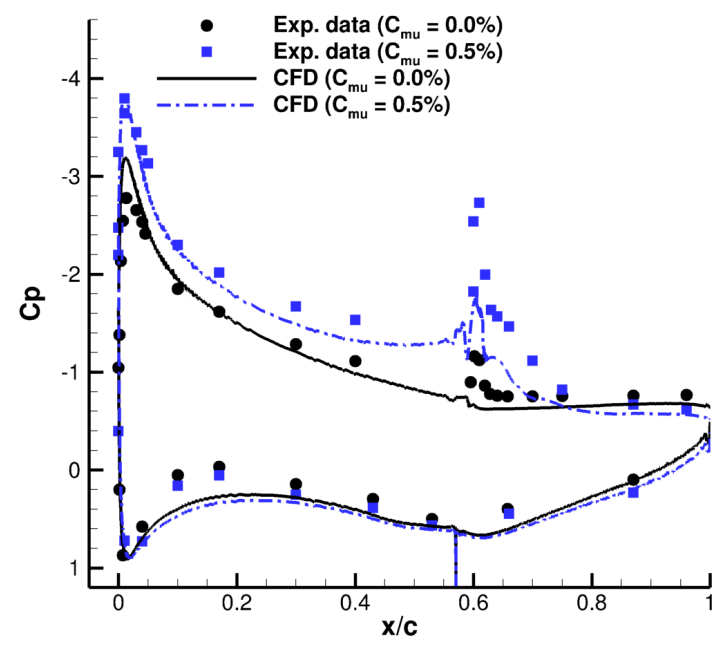

(c) outboard, $z / b=89 \%$

Figure 10. Chordwise surface pressure comparisons: baseline case, $\delta_{R}=30^{\circ}, \beta=0^{\circ}$. 


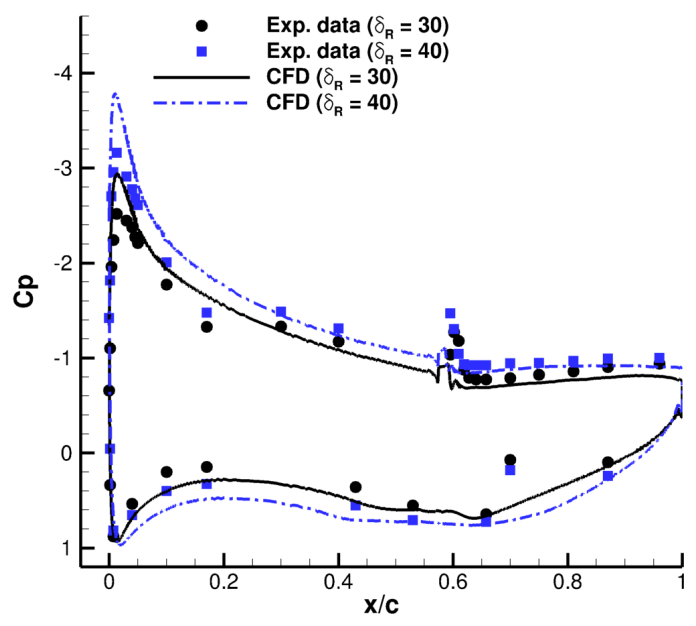

(a) $C_{m u}=0.0 \%$

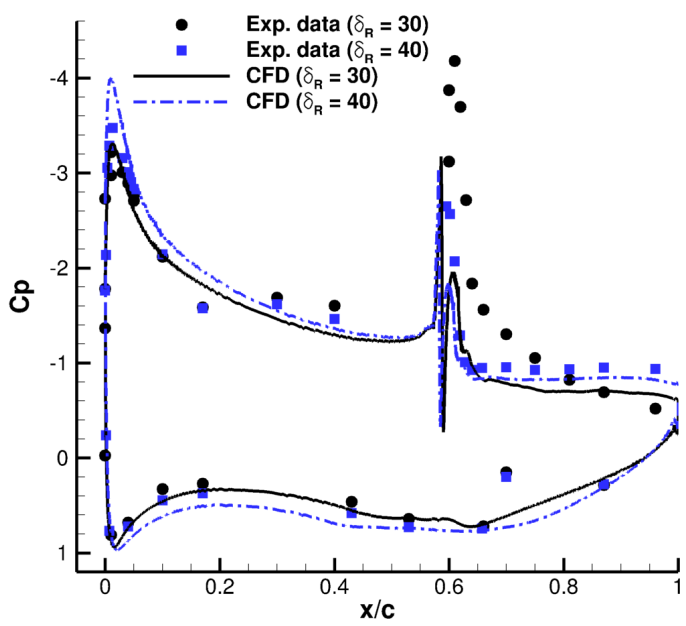

(b) $C_{m u}=0.5 \%$

Figure 11. Effect of varying rudder deflection on chordwise pressures at $\mathbf{z} / \mathbf{b}=\mathbf{7 0} \%$ section, $\beta=0^{\circ}$.

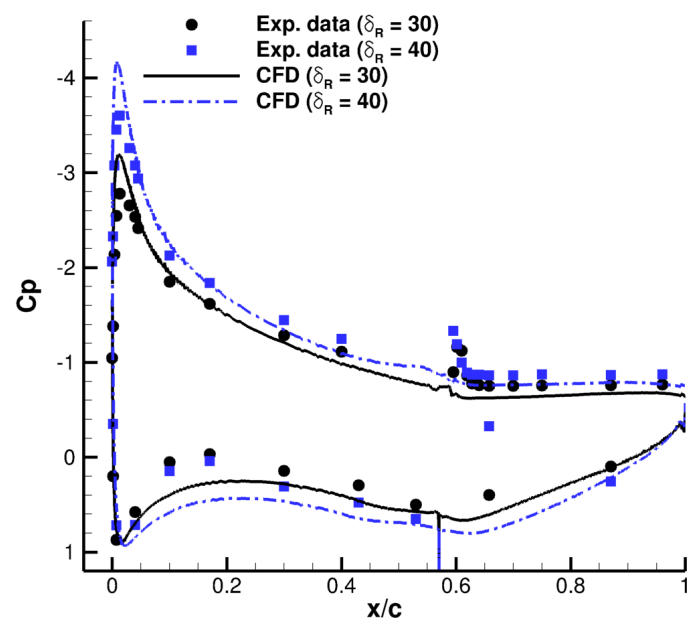

(a) $C_{m u}=0.0 \%$

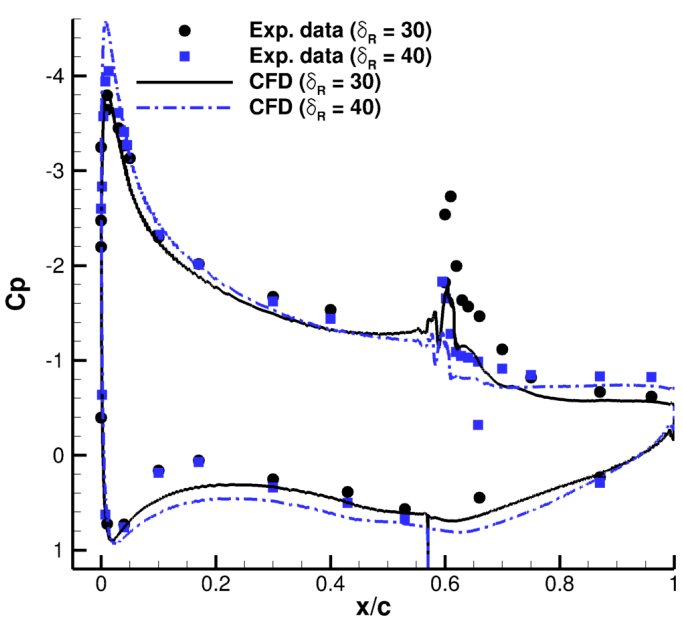

(b) $C_{m u}=0.5 \%$

Figure 12. Effect of varying rudder deflection on chordwise pressures at $\mathbf{z} / \mathbf{b}=89 \%$ section, $\beta=0^{\circ}$. 

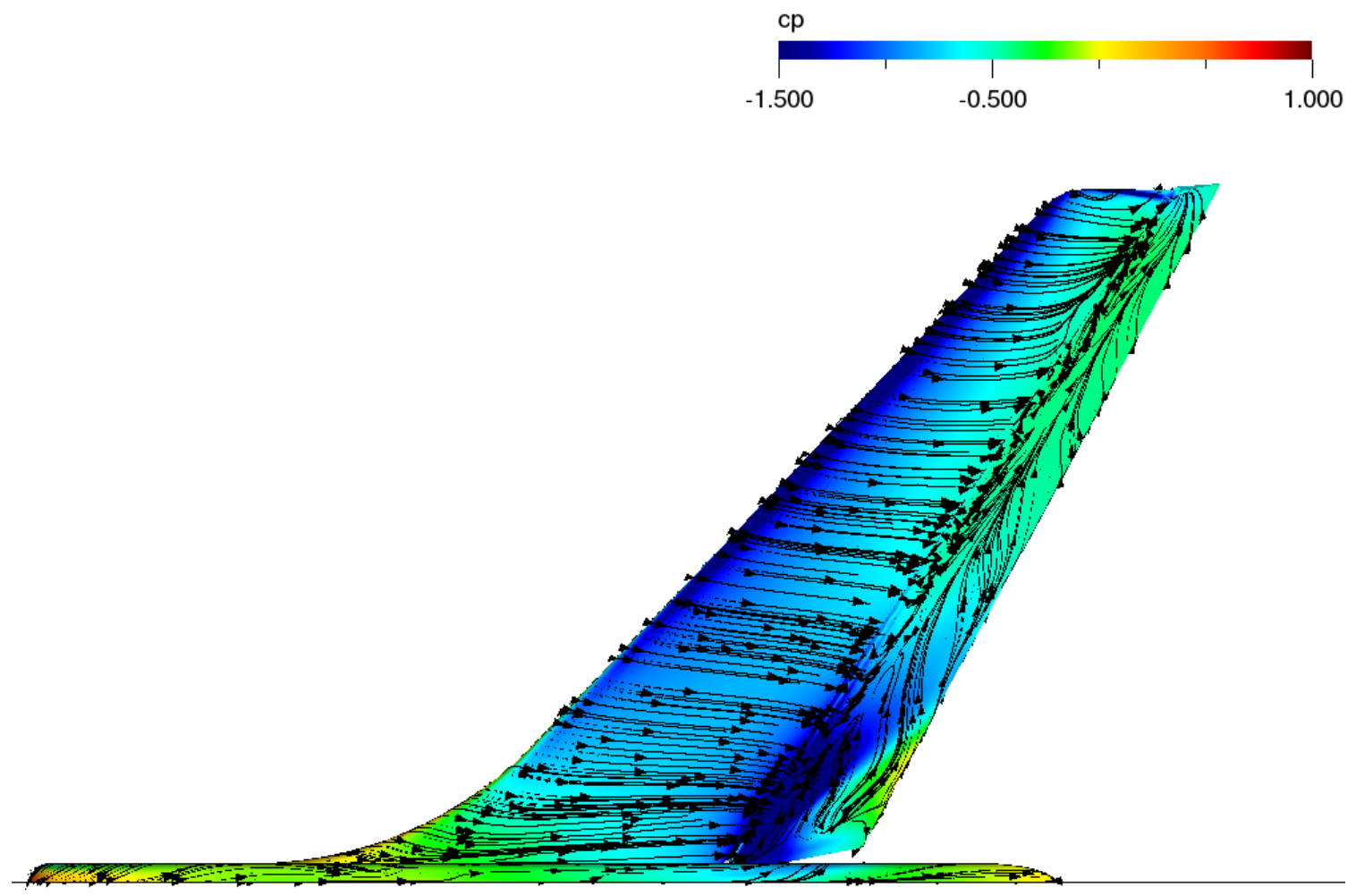

(a) $C_{m u}=0.0 \%$

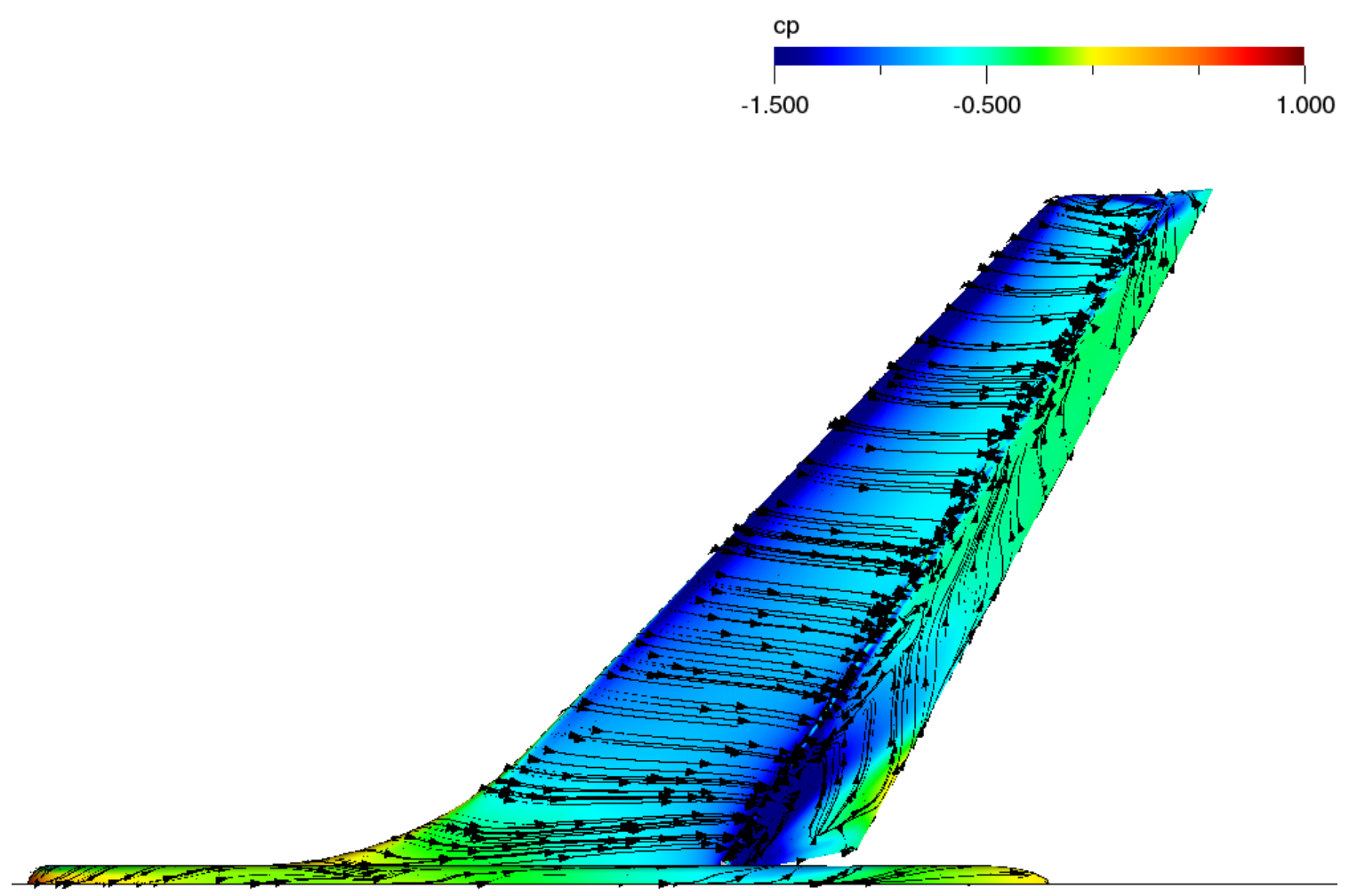

(b) $C_{m u}=0.5 \%$

Figure 13. Effect of actuation on simulated suction surface streamlines, $\delta_{R}=40^{\circ}, \beta=0^{\circ}$ 


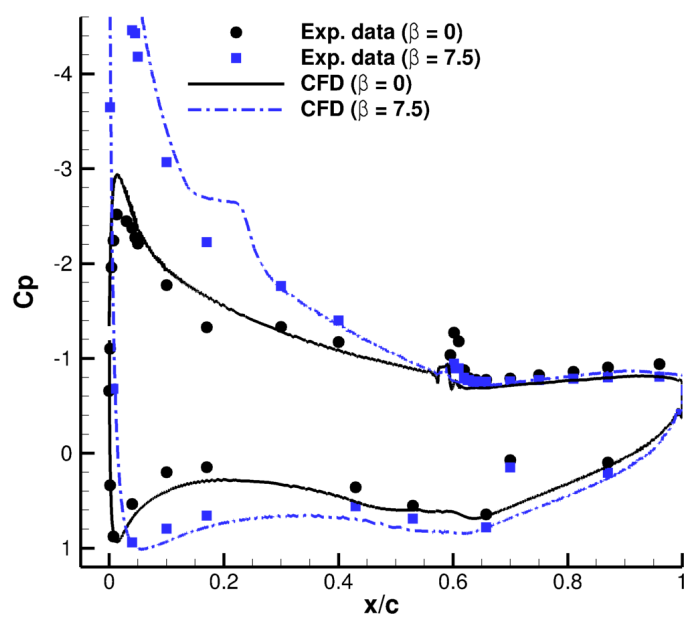

(a) $C_{m u}=0.0 \%$

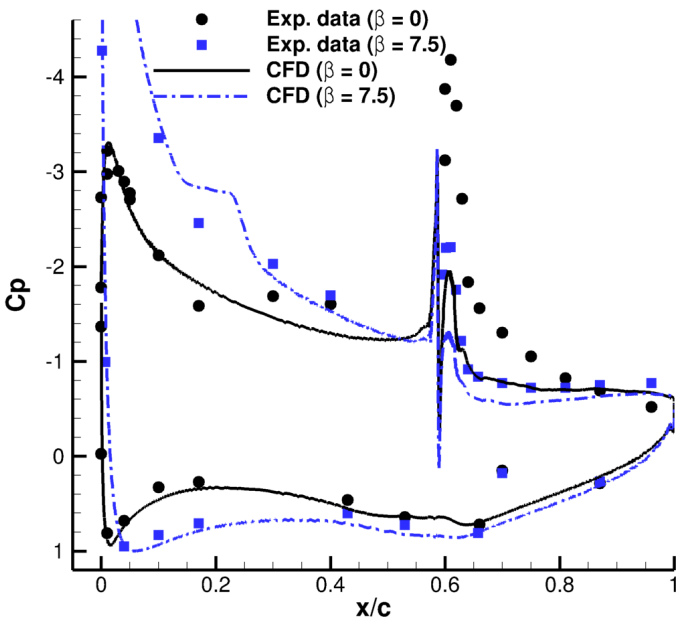

(b) $C_{m u}=0.5 \%$

Figure 14. Effect of varying yaw angle on chordwise pressures at $\mathbf{z} / \mathbf{b}=70 \%$ section, $\delta_{R}=30^{\circ}$.

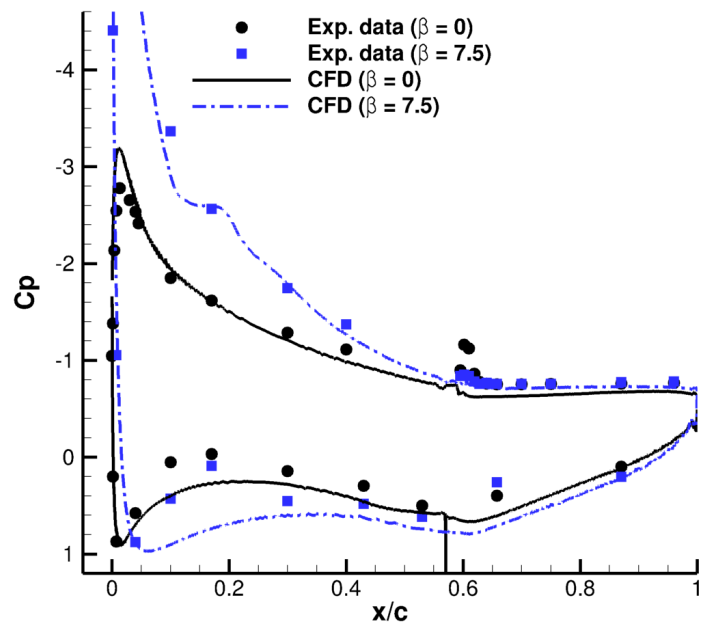

(a) $C_{m u}=0.0 \%$

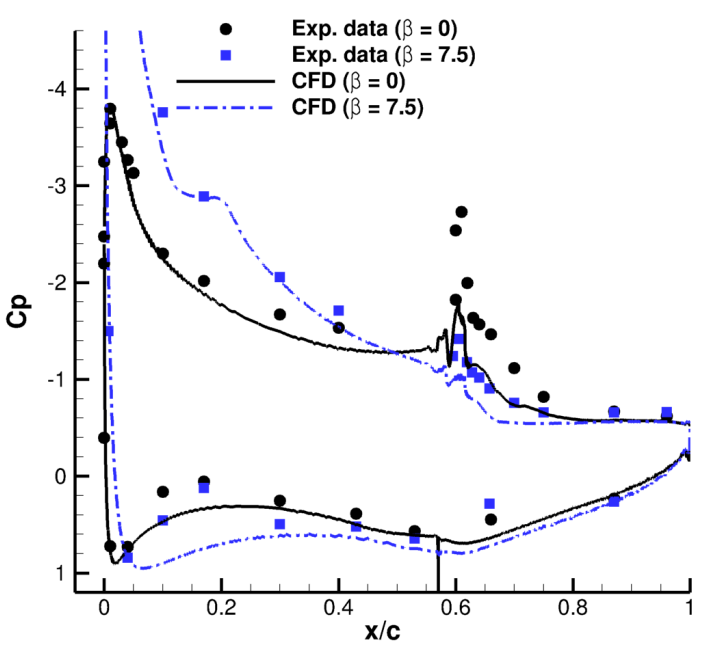

(b) $C_{m u}=0.5 \%$

Figure 15. Effect of varying yaw angle on chordwise pressures at $\mathbf{z} / \mathbf{b}=89 \%$ section, $\delta_{R}=30^{\circ}$. 


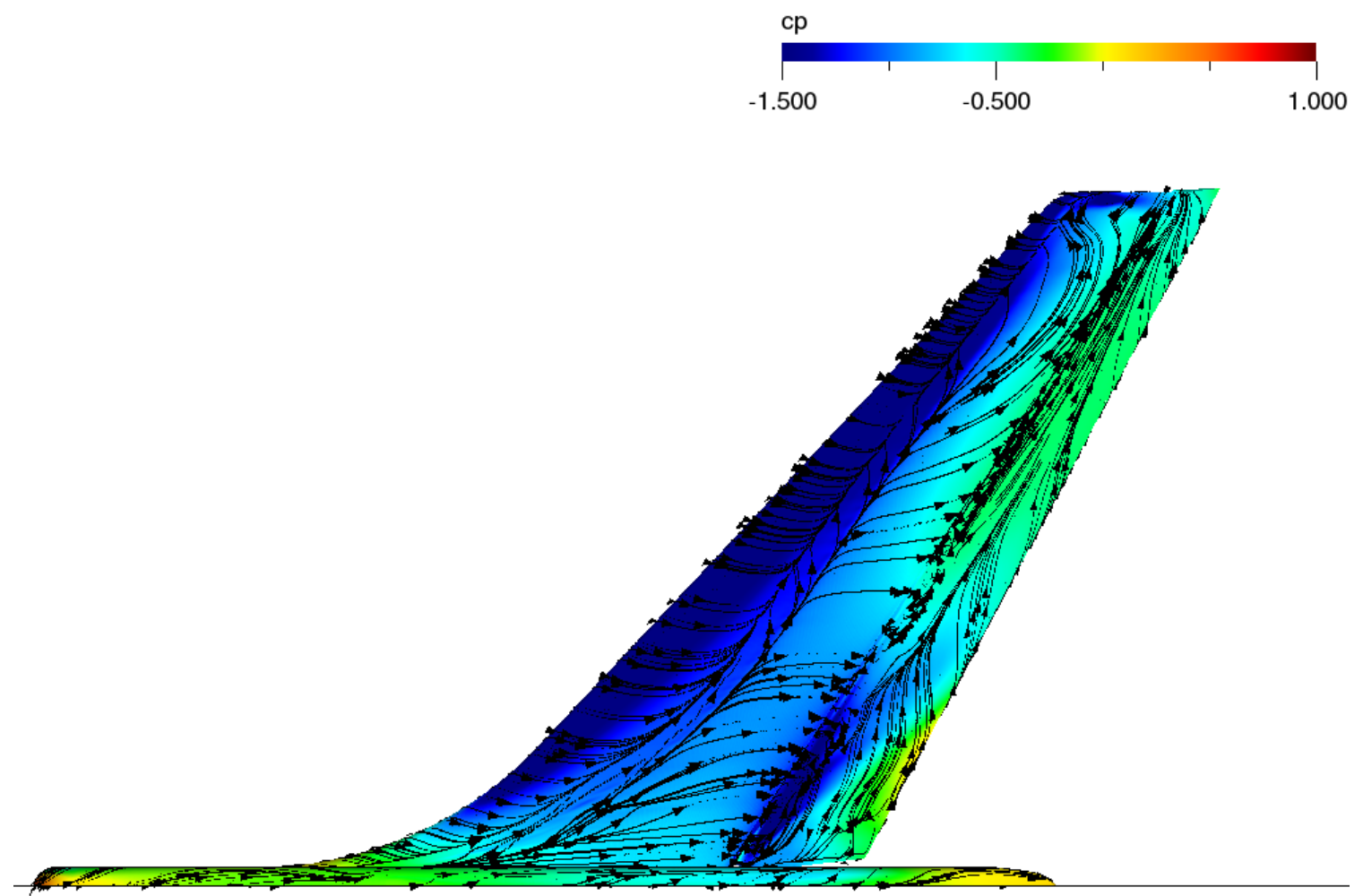

(a) $C_{m u}=0.0 \%$
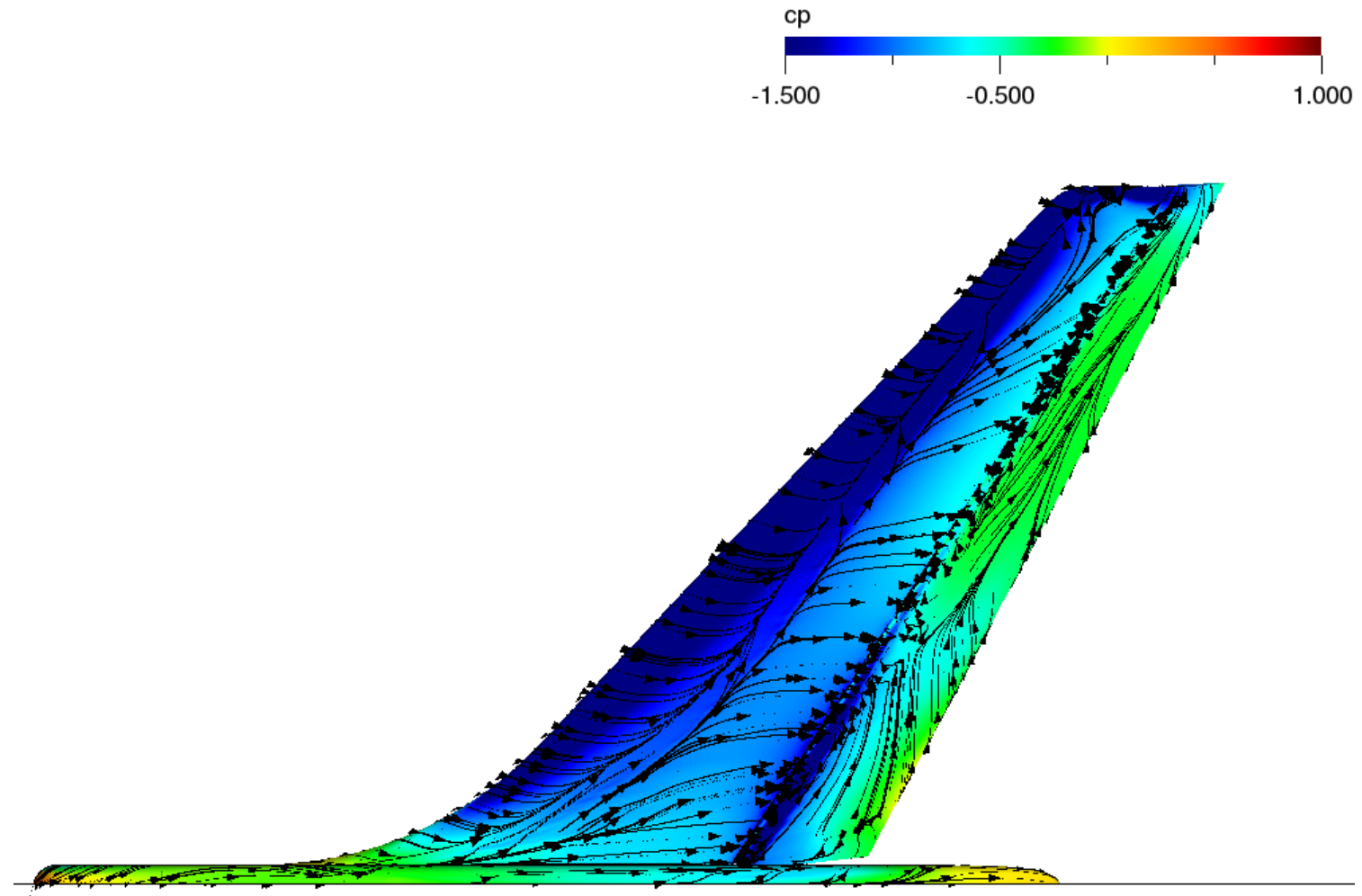

(b) $C_{m u}=0.5 \%$

Figure 16. Effect of actuation on simulated suction surface streamlines, $\delta_{R}=30^{\circ}, \beta=7.5^{\circ}$. 


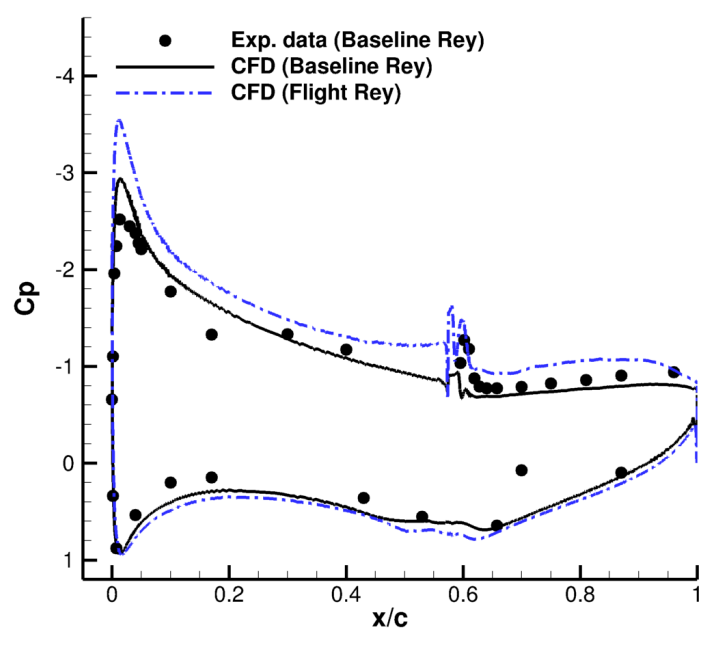

(a) $C_{m u}=0.0 \%$

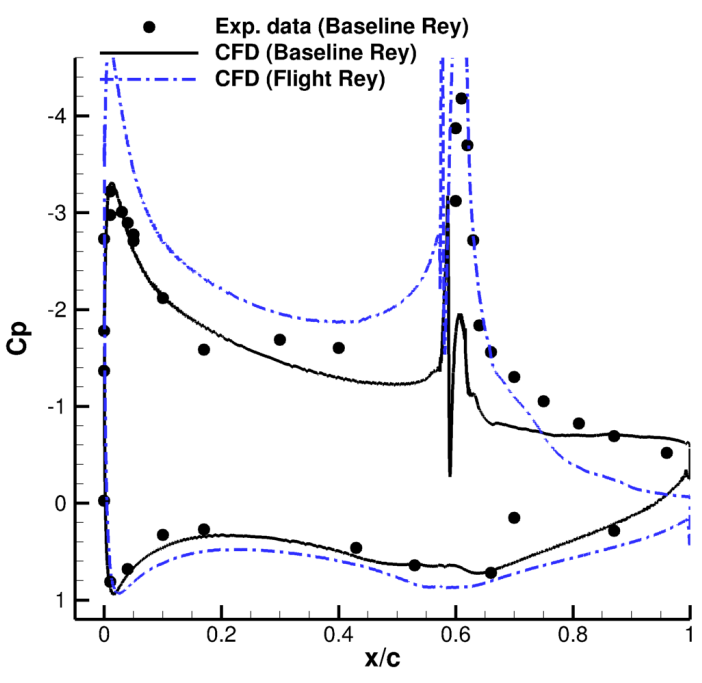

(b) $C_{m u}=0.5 \%$

Figure 17. Effect of varying Reynolds number on chordwise pressures at $\mathbf{z} / \mathbf{b}=\mathbf{7 0 \%}$ section, $\delta_{R}=30^{\circ}, \beta=0^{\circ}$.

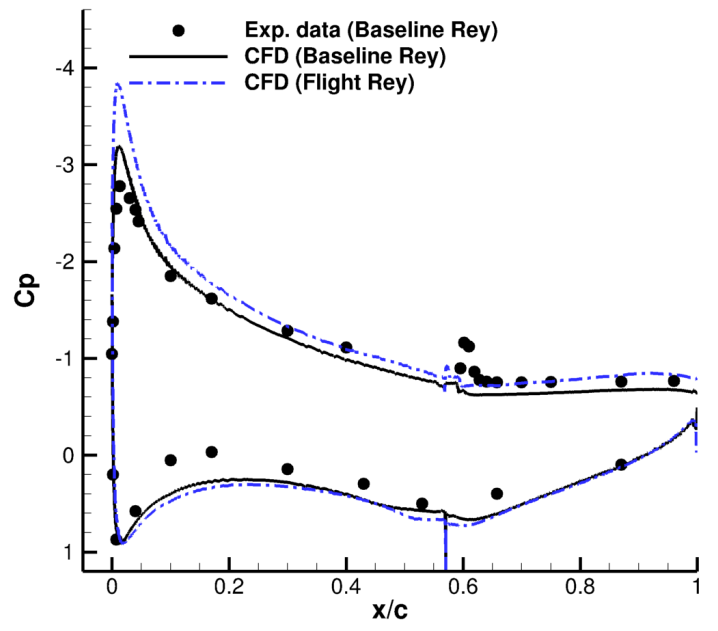

(a) $C_{m u}=0.0 \%$

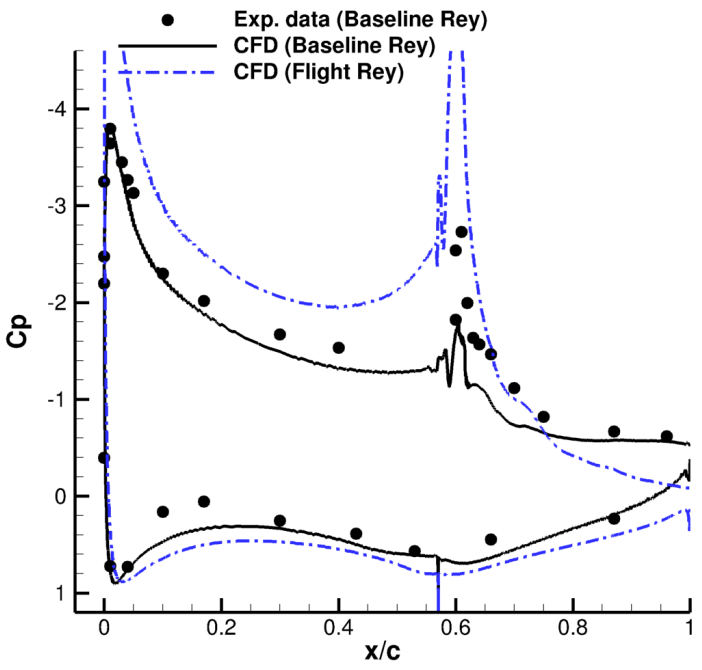

(b) $C_{m u}=0.5 \%$

Figure 18. Effect of varying Reynolds number on chordwise pressures at $\mathbf{z} / \mathbf{b}=\mathbf{8 9 \%}$ section, $\delta_{R}=30^{\circ}, \beta=0^{\circ}$. 


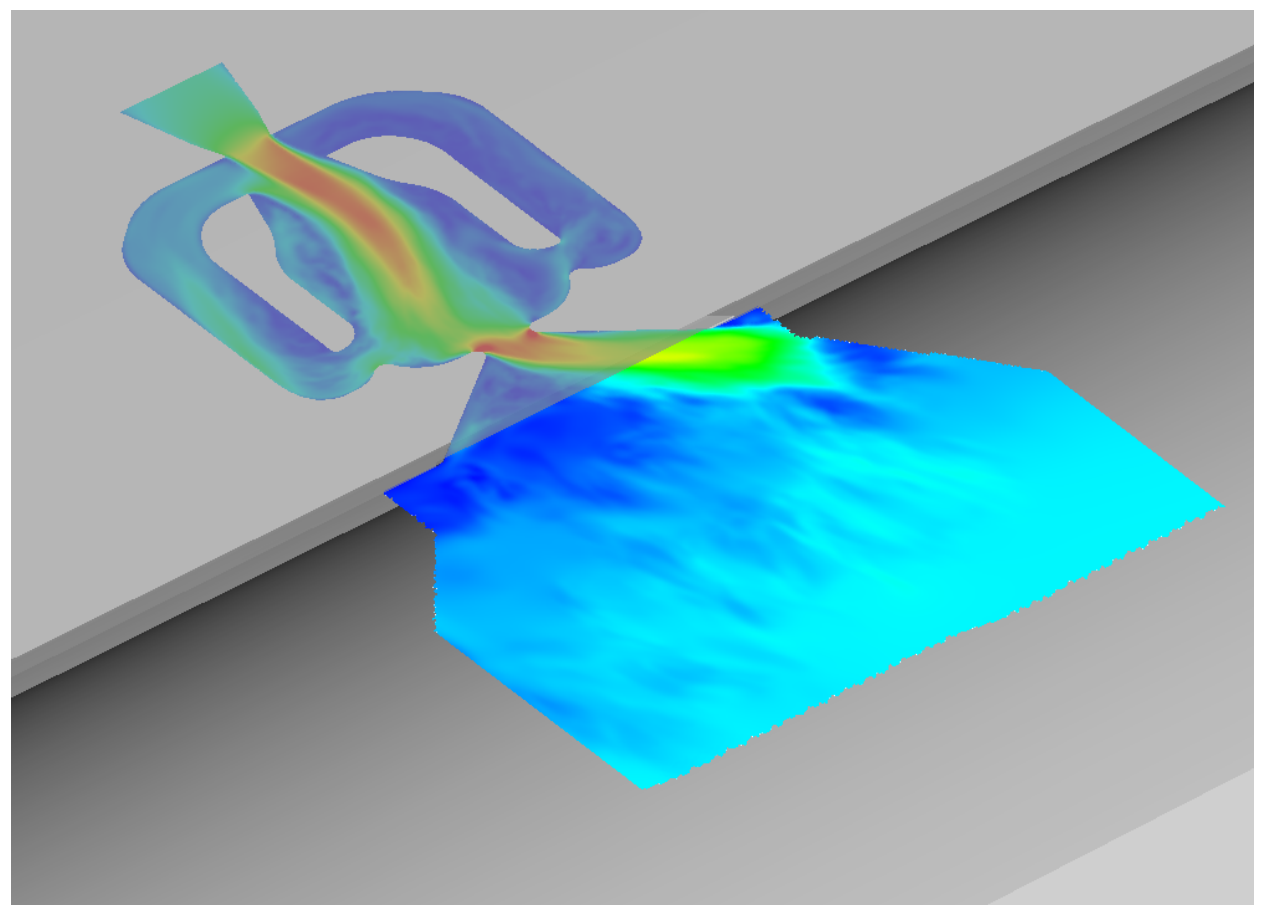

(a) Frame 1

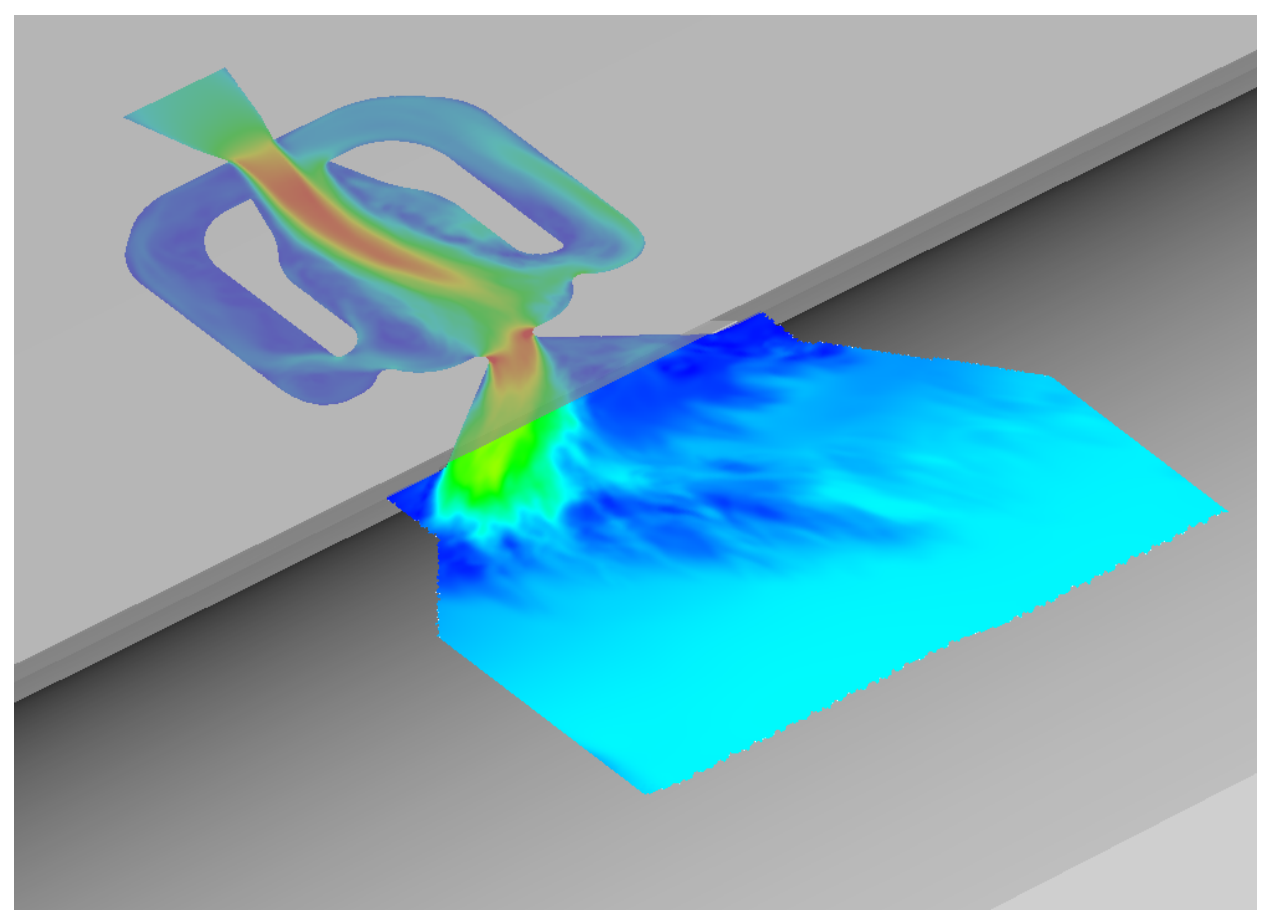

(b) Frame 2

\section{Velocity Magnitude $[\mathrm{m} / \mathrm{sec}]$}

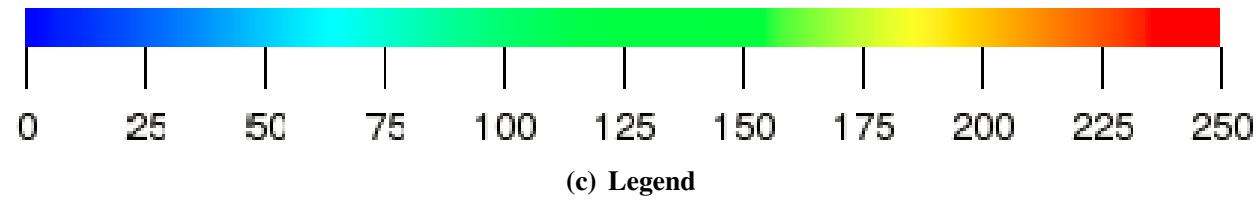

Figure 19. Velocity contours for actuator \# 7 at two different times, baseline configuration. 